\title{
sFRP2 in the aged microenvironment drives melanoma metastasis and therapy resistance
}

\author{
Amanpreet Kaur ${ }^{1,2}$, Marie R. Webster ${ }^{1}$, Katie Marchbank ${ }^{1}$, Reeti Behera ${ }^{1}$, Abibatou Ndoye ${ }^{1}$, \\ Curtis H. Kugel III ${ }^{1}$, Vanessa M. Dang ${ }^{1}$, Jessica Appleton ${ }^{1}$, Michael P. O'Connell ${ }^{1}$, Phil \\ Cheng $^{3}$, Alexander A. Valiga1 ${ }^{1}$, Rachel Morissette ${ }^{4}$, Nazli B. McDonnell ${ }^{4}$, Luigi Ferrucci ${ }^{4}$, \\ Andrew V. Kossenkov ${ }^{1}$, Katrina Meeth ${ }^{5}$, Hsin-Yao Tang ${ }^{1}$, Xiangfan Yin ${ }^{1}$, William H. Wood \\ III $^{4}$, Elin Lehrmann ${ }^{4}$, Kevin G. Becker ${ }^{4}$, Keith T. Flaherty ${ }^{6}$, Dennie T. Frederick ${ }^{6}$, Jennifer A. \\ Wargo $^{7}$, Zachary A. Cooper ${ }^{7}$, Michael T. Tetzlaff ${ }^{7}$, Courtney Hudgens ${ }^{7}$, Katherine M. Aird ${ }^{1}$, \\ Rugang Zhang ${ }^{1}$, Xiaowei Xu ${ }^{8}$, Qin Liu ${ }^{1}$, Edmund Bartlett ${ }^{8}$, Giorgos Karakousis ${ }^{8}$, Zeynep \\ Eroglu $^{9}$, Roger S. Lo ${ }^{10}$, Matthew Chan ${ }^{11}$, Alexander M. Menzies ${ }^{12}$, Georgina V. Long ${ }^{12}$, \\ Douglas B. Johnson ${ }^{13}$, Jeffrey Sosman ${ }^{13}$, Bastian Schilling ${ }^{14}$, Dirk Schadendorf ${ }^{14}$, David \\ W. Speicher ${ }^{1}$, Marcus Bosenberg ${ }^{5}$, Antoni Ribas ${ }^{10}$, and Ashani T. Weeraratna ${ }^{1}{ }^{* *}$
}

${ }^{1}$ The Wistar Institute, Philadelphia, Pennsylvania ${ }^{2}$ University of the Sciences, Philadelphia, Pennsylvania ${ }^{3}$ University of Zurich, Zurich, Switzerland ${ }^{4}$ The National Institute on Aged, NIH, Baltimore, Maryland ${ }^{5}$ Yale University, New Haven, Connecticut ${ }^{6}$ Massachusetts General Hospital Cancer Center, Boston, Massachusetts ${ }^{7} \mathrm{MD}$ Anderson Cancer Center, Houston, TX ${ }^{8}$ Abramson Cancer Center, University of Pennsylvania, Philadelphia, Pennsylvania ${ }^{9}$ Department of Medical Oncology, City of Hope Medical Center, Duarte, CA ${ }^{10}$ Department of Medicine, Division of Hematology-Oncology, University of California Los Angeles, Los Angeles, California (UCLA) ${ }^{11}$ Crown Princess Mary Cancer Centre, Westmead Hospital, Westmead, Australia ${ }^{12}$ Melanoma Institute Australia and The University of Sydney, Sydney, Australia ${ }^{13}$ Vanderbilt University Medical Center, Nashville TN ${ }^{14}$ Department of Dermatology, University Hospital, West German Cancer Center, University Duesburg-Essen, Essen, Germany and German Cancer Consortium (DKTK), Heidelberg, Germany

\section{Abstract}

Users may view, print, copy, and download text and data-mine the content in such documents, for the purposes of academic research, subject always to the full Conditions of use:http://www.nature.com/authors/editorial_policies/license.html\#termsReprints: Reprints and permissions information is available at www.nature.com/reprints

${ }^{\star *}$ To Whom Correspondence Should Be Addressed: Ashani T. Weeraratna, Ph.D., The Wistar Institute, Rm 452/454A, 3601 Spruce Street, Philadelphia, PA 19104, Office: 215 495-6937, Fax: 215 495-6938, ; Email: aweeraratna@wistar.org

Author Contributions

ATW conceived and designed the project. ATW and AK designed and supervised the experiments. AK, MRW, KM, RB, AN, CHKIII, VMD, JA, MPO, PC, AAV, WHWIII, EL and KMA performed the experiments. ATW, AK, AVK, HYT, XY, EL, ZE, KGB, RZ, XX, QL and DWS analysed the experimental data. ATW, AK and QL designed and supervised data analysis and statistical analysis. MB, AR, DS, JS, BS, RSL, MC, AMM, GVL, DBJ, RM, NBM, LF, KM, KTF, DTF, JAW, ZAC, MTT, CH, EB, GK, performed data collection and provided anonymized patient data and samples and reagents. ATW and AK wrote the manuscript. All authors discussed the results and commented on the manuscript.

Data Deposition: Microarray data are available in the GEO database (accession \# GSE57445).

Competing Financial Interests: Keith Flaherty is a consultant to: GlaxoSmithKline, Roche, and Novartis; Georgina Long is a consultant to: Amgen, BMS, GSK, MSD, Novartis, Roche; Jennifer Wargo is a consultant to: Roche and GSK. We do not believe these relationships have any direct impact on this work. 
Cancer is a disease of aging, and aged cancer patients have a poorer prognosis. This may be due to accumulated cellular damage, decreases in adaptive immunity, and chronic inflammation.

However, the effects of the aged microenvironment on tumor progression have been largely unexplored. Since dermal fibroblasts can have profound impacts on melanoma progression ${ }^{1-4}$ we examined whether age-related changes in dermal fibroblasts could drive melanoma metastasis and response to targeted therapy. We find that aged fibroblasts secrete a Wnt antagonist, sFRP2, which activates a multi-step signaling cascade in melanoma cells that results in a decrease in $\beta$-catenin and MITF, and ultimately the loss of a key redox effector, APE1. Loss of APE1 attenuates the response of melanoma cells to ROS-induced DNA damage, rendering them more resistant to targeted therapy (vemurafenib). Age-related increases in sFRP2 also augment both angiogenesis and metastasis of melanoma cells. These data provide an integrated view of how fibroblasts in the aged microenvironment contribute to tumor progression, offering new paradigms for the design of therapy for the elderly.

To determine whether the aged microenvironment promotes melanoma progression, Yumm1.7 cells, derived from the $\mathrm{Braf}^{\mathrm{V} 600 \mathrm{E}} /{\mathrm{Cdkn} 2 \mathrm{a}^{-/-} / \mathrm{Pten}^{-/-} \text {mouse model of melanoma, }}^{5}$, were injected into 8-week old mice (young) or 52-week old mice (aged). Tumors grow slowly in aged mice (Figure 1a), but are more aggressive, with increased angiogenesis (Figure 1b) and lung metastases (Figure 1c, Extended Data 1a). This is consistent with observations that melanoma cells switch between proliferative and invasive states, termed "phenotype switching", which depends on changes in Wnt signaling and $\operatorname{MITF}^{6,7,}$, . We have shown that phenotype switching to a metastatic, therapy-resistant state is induced by stresses such as hypoxia ${ }^{9}$.

Cross-talk between dermal fibroblasts and transformed melanocytes is critical for melanoma invasion ${ }^{14}{ }^{4}$ and therapy resistance ${ }^{10}$. Dermal fibroblasts senesce during aging ${ }^{11}$, and senescent fibroblasts can promote tumor invasion ${ }^{12}$. To determine whether fibroblasts from normally-aged skin could promote tumor progression, we used dermal fibroblasts from young $(<35)$ and aged $(>55)$ healthy donors from the Baltimore Longitudinal Study of Aging ${ }^{13}$ (Extended Data Table 1). Proliferation is initially unaffected (Extended Data 1b), but aged fibroblasts senesce more rapidly than young fibroblasts (Extended Data 1c). Organotypic skin reconstructs were built using two melanoma cell lines (WM35 and WM793) and 3 young or 3 aged fibroblast lines, which persisted equally in the skin reconstructs as demonstrated by smooth muscle actin staining (Extended Data 1d).

Melanoma cell invasion was increased, but proliferation was decreased in reconstructs built with aged fibroblasts (Figure 1d, e, Extended Data 1e). To assess the effects of secreted factors, melanoma cells were exposed to conditioned media from aged or young fibroblasts. Aged fibroblast media decreased melanoma cell proliferation (Figure 1f, Extended Data 1f), and increased invasion as measured by spheroid and Boyden chamber invasion assays (Figure 1g,h Extended Data 1g). These data indicate that secreted factors from fibroblasts play critical roles in phenotype switching in melanoma cells.

A key player in phenotype switching is $\beta$-catenin, which drives proliferation and inhibits invasion of melanoma cells ${ }^{14}, 15$. An inhibitor of $\beta$-catenin, sFRP2, was significantly increased in the secretome of aged fibroblasts (Figure 2a). In both spheroid and Boyden 
Chamber assays, media from young fibroblasts treated with recombinant sFRP2 (rsFRP2) increased invasion of melanoma cells (Figure 2b, Extended Data 2a) and media from aged fibroblasts treated with a-sFRP2 inhibited melanoma cell invasion (Figure 2c, Extended Data 2c). In vivo, sFRP2 was increased in sera from aged mice, and these levels could be nearly attained in young mice by intravenous injection of rsFRP2 (Figure 2d). Increasing serum sFRP2 in young mice increased metastasis of Yumm1.7 to the lung (Figure 2e) and increased angiogenesis in subcutaneous tumors (Figure 2f, Extended Data 2c,d). Tumors in a-sFRP2 treated mice showed impaired angiogenesis (Figure 2f, Extended Data 2e,f). However, a-sFRP2 treated aged mice succumbed to a lethal inflammation (Extended Data 3 ), an outcome previously observed in aged immune-competent mice treated with a-PD $1^{16}$.

We next explored the significance of sFRP2 effects on $\beta$-catenin expression and activity. Treatment of melanoma cells with rsFRP2 inhibited $\beta$-catenin expression (Figure 2g), and $\beta$ catenin was decreased in aged human skin (Figure 2h, Extended Data 4a) and in melanoma cells treated with aged fibroblast media (Extended Data 4b,c). Yumm1.7 tumors in aged mice exhibited decreased $\beta$-catenin and increased sFRP2 (Figure 2i), effects reversed by treatment of aged mice with $\alpha$-sFRP2 (Figure $2 \mathrm{j}$ ). Knockdown of $\beta$-catenin in young fibroblasts increased their sFRP2 secretion (Figure $2 \mathrm{k}$ ), which reduced $\beta$-catenin and increased invasion in melanoma cells (Figure 21, Extended Data 4d). These data suggest that sFRP2 present in the aged microenvironment may contribute to melanoma metastasis through suppression of $\beta$-catenin in melanoma cells.

The loss of $\beta$-catenin permits oxidative stress in hematopoietic stem cells ${ }^{17}$. We hypothesized that the same might occur in melanoma cells, via MITF, which is activated by $\beta$-catenin ${ }^{18}$. MITF modulates responses to reactive oxygen species (ROS) by increasing the redox effector APE1 (REF-1) ${ }^{19}$. We predicted that age-induced loss of $\beta$-catenin might dysregulate the MITF/APE1 redox pathway. Indeed, melanoma cells exposed to aged fibroblast media have decreased $\beta$-catenin, MITF, and APE1 (Figure 3a). APE1 expression is lower in aged human skin (Extended Data 5a), and in Yumm1.7 tumors in aged mice (Figure 3b). Loss of APE1 reduces cellular responses to ROS. ROS activity is increased in aged fibroblasts (Figure 3c), partly due to the fact that aged fibroblasts secrete lower levels of superoxide dismutase 3 (SOD3), and peroxiredoxin 6, which scavenge free radicals (Extended Data 5b,c). A marker of oxidative stress, 8-oxo-dG, was increased in aged but not young skin (Extended Data 5d) and in tumors in aged mice (Figure 3d). Knockdown of APE1 in melanoma cells increases ROS activity, especially upon exposure to aged fibroblast media (Figure 3e,f, Extended Data 5e). We hypothesized that this loss of ability to respond to ROS in an aged environment is partly due to SFRP2 downregulation of APE1. Treating young mice with rsFRP2 increases 8-oxo-dG in Yumm1.7 tumors (Figure 3g), while depleting sFRP2 in aged mice decreases 8-oxo-dG (Figure 3g). Together, these data indicate that sFRP2 increases oxidative stress by inhibiting $\beta$-catenin and, ultimately, APE1 in melanoma cells (Figure 3h).

Our model indicates that loss of APE1 due to age-induced sFRP2 should render melanoma cells sensitive to DNA damage (Extended Data 6a). Accordingly, microarray analysis reveals an increased DNA damage response signature in melanoma cells exposed to aged fibroblasts (Extended Data 6b,c). The DNA damage markers $\gamma \mathrm{H} 2 \mathrm{AX}$ and 53BP1 are increased in 
melanoma cells grown in aged mice (Figure 4a), or exposed to aged fibroblast media (Figure $4 b$ ) or in skin reconstructs built with aged fibroblasts (Extended Data 7a,b). Melanoma cells exposed to aged media also had increased levels of DNA damage (Figure 4c, Extended Data 7c). Pre-treating aged fibroblasts with the anti-oxidant $\mathrm{N}$-acetylcysteine (NAC) decreased ROS (Extended Data 7d) and, subsequently, $\gamma \mathrm{H} 2 \mathrm{AX}$ in melanoma cells (Figure 4d). Conversely, knockdown of the anti-oxidant SOD3 in young fibroblasts increased $\gamma \mathrm{H} 2 \mathrm{AX}$ in melanoma cells (Figure 4e, Extended Data 7e), suggesting that age-induced DNA damage could be reversed by inhibiting ROS activity.

We next queried the contribution of the sFRP2 $\rightarrow$ APE1 signaling axis to ROS-induced DNA damage. Loss of APE1 increases $\gamma \mathrm{H} 2 \mathrm{AX}$ (Figure 4f). In melanoma cells, $\gamma \mathrm{H} 2 \mathrm{AX}$ also increases with treatment of rsFRP2, and decreases in cells where sFRP2 is deleted (Figure 4g, Extended Data 7f,g). In vivo, 53BP1 increases in Yumm1.7 tumors in young mice treated with rsFRP2, and decreases in aged mice treated with a-sFRP2 (Figure 4h). Aged melanoma patients (over 55) had significantly higher serum levels of sFRP2 ( $\mathrm{p}=0.0084)$ than young patients (under 40) (Figure 4i). The key players in the pathway outlined in Figure $3 \mathrm{~h}$ were altered in aged melanoma patients: increased SFRP2 (in both fibroblasts and melanoma cells), decreased $\beta$-catenin, MITF and APE1, and increased 8-oxo-dG and 53BP1 (Figure 4j, Extended Data 8). These data confirmed our observations from both human cell line and mouse data.

Increases in $\operatorname{ROS}^{20,21}$ and decreases in $\beta$-catenin ${ }^{9,2}$ and MITF $^{23}$ have been associated with increased resistance to BRAF inhibitors. Accordingly, spheroids treated with young medium were more sensitive to PLX4720 (vemurafenib) than those exposed to aged medium (Extended Data 9a). In vivo, Yumm1.7 tumors in young mice responded to PLX4720 more robustly than tumors in aged mice (Figure 5a,b). Melanoma cells first exposed to media from young fibroblasts treated with $\mathrm{H}_{2} \mathrm{O}_{2}$ (to increase ROS), developed resistance to PLX4720 (Figure 5c). However, when we first exposed melanoma cells to media from aged fibroblasts pre-treated with NAC, the melanoma cells died, whether treated with NAC alone or NAC + PLX4720 (Figure 5d, e). This indicated that vemurafenib-resistant cells in an aged microenvironment might be uniquely sensitive to anti-oxidants. This finding is especially exciting in light of recent data that indicate that anti-oxidants are effective in treating KRAS and BRAF mutant colon cancers ${ }^{24}$.

Phenotype switching resulting in the loss of $\beta$-catenin has also been shown to contribute to resistance to vemurafenib ${ }^{9}, 22$. $\beta$-catenin expression is elevated in sensitive vs. resistant cell lines (Extended Data 9b). Further, patients with clinical response to vemurafenib (over 30\% tumor reduction as measured by RECIST) had higher levels of $\beta$-catenin than those with muted response (Figure 5f, g). Yumm1.7 cells are sensitive to PLX4720, and knocking down Ctnnb1 in these cells significantly increased their resistance to PLX4720 (Extended Data 9c). To determine whether sFRP2 alters the response of melanoma cells to vemurafenib, rsFRP2 was administered i.v. to tumor-bearing young mice, prior to, and during PLX4720 exposure. $\beta$-catenin was decreased in the skin of sFRP2-treated mice (Extended Data 9d), and treated mice rapidly developed resistance to PLX4720 (Figure 5h). Depletion of sFRP2 in aged mice began to re-sensitize tumors to PLX4720 (Figure 5i, Extended Data 9e), but a 
firm conclusion cannot be drawn from these data due to the premature death of the mice as described earlier.

Since these data suggested that response to BRAF inhibitors may be attenuated in aged patients, we compared the age of patients $(n=79)$ at starting treatment to percent tumor reduction (RECIST) post-vemurafenib. Using age categories of under 35 and over 55, we saw a shift from a $41 \%$ (mean) response by RECIST in young patients to $25 \%$ (mean) response in patients over 55. Numbers of young patients were too low to achieve statistical significance. We therefore refocused our efforts on identifying an age cutoff where a statistically significant difference could be observed. We observed a striking separation $(\mathrm{p}=0.016)$ between patients under 65 yo and over 65 yo ( $25.6 \%$ reduction in tumor burden in aged compared to $\sim 47 \%$ in the young, Figure $5 \mathrm{j}$ ). The continous relationship between response and age was highly significant (Spearman's correlation coefficient of $r=0.243$, $\mathrm{p}=0.03$ ) (Extended Data 9f). However, in this small sample set, the relationship between patient age, sFRP2 and RECIST was not significant. Together, these data suggest that sFRP2-induced $\beta$-catenin loss not only promotes invasion, but also renders melanoma cells more senstive to oxidative stress. New data from Cantley and colleagues show that therapyrefractory, BRAF mutant cells are uniquely sensitive to anti-oxidants, specifically Vitamin $\mathrm{C}^{24}$. Our data support these observations, and suggest that aged patients may uniquely benefit from anti-oxidant therapy.

In our experiments, genetically identical cells had a very different outcome in terms of metastasis and therapy response when placed in an aged microenvironment. In the skin, benign melanocytic lesions (nevi) bear mutational changes that do not result in tumorigenesis, until cells accumulate further changes during aging. These changes may be genetic, such as loss of p16, but they may also be epigenetic, and age-related epigenetic changes also contribute to tumorigenesis ${ }^{25} 27$. What drives the initiation of these epigenetic changes is not well understood, and it is possible that the aged secretome contributes to this process. While overall results were consistent among different fibroblasts of similar ages, we did observe some phenotypic differences. These may be attributable to factors such as tanning, as indeed, the exposure of melanoma cells to UV irradiation not only drives tumor promotion $^{28}$, but also metastasis ${ }^{29}$. Our data suggest that, as the general population ages, new efforts must be made to understand and treat cancer in an age-appropriate manner.

\section{METHODS}

\section{Cell Culture}

FS5, FS4, FS13, FS14, M93-047, UACC-903 and UACC-1273 cells were maintained in RPMI (Invitrogen, Carlsbad, CA), supplemented with 10\% FBS, 100 units $/ \mathrm{ml}$ penicillin and streptomycin and $4 \mathrm{mM} \mathrm{L-glutamine.} \mathrm{WM35,} \mathrm{WM793,} \mathrm{WM164,} \mathrm{WM1799} \mathrm{and} \mathrm{1205LU}$ cells were maintained in MCDB153 (Sigma, St Louis, MO)/ L-15 (Cellgro, Manassas, VA) (4:1 ratio) supplemented with $2 \% \mathrm{FBS}$ and $1.6 \mathrm{mM} \mathrm{CaCl}_{2}$ (Tumor growth media). WM983b, WM3918 cells were maintained in DMEM (Invitrogen, Carlsbad, CA), supplemented with 5\% FBS, 100 units/ml penicillin and streptomycin and $4 \mathrm{mM} \mathrm{L-}$ glutamine. YUMM1.7 cells were maintained in DMEM F-12 (Hepes/glutamine) supplemented with $10 \%$ FBS, $1 \%$ NEAA, and 100 units $/ \mathrm{ml}$ penicillin and streptomycin. 
Fibroblasts were maintained in DMEM, supplemented with 10\% FBS, 100 units $/ \mathrm{ml}$ penicillin and streptomycin and $4 \mathrm{mM} \mathrm{L}$-glutamine. Keratinocytes were maintained in keratinocyte SFM supplemented with human recombinant Epidermal Growth Factor 1-53 (EGF 1-53) and Bovine Pituitary Extract (BPE) (Invitrogen). Cell lines were cultured at $37^{\circ} \mathrm{C}$ in $5 \% \mathrm{CO}_{2}$ and the medium was replaced as required. Cell stocks were fingerprinted using AmpFLSTR $®$ Identifiler ${ }^{\circledR}$ PCR Amplification Kit from Life Technologies TM at The Wistar Institute Genomics Facility. Although it is desirable to compare the profile to the tissue or patient of origin, our cell lines were established over the course of 40 years, long before acquisition of normal control DNA was routinely performed. However, each STR profile is compared to our internal database of over 200 melanoma cell lines, as well as control lines, such as HeLa and 293T. STR profiles are available upon request. Cell culture supernatants were mycoplasma tested using a Lonza MycoAlert assay at the University of Pennsylvania Cell Center Services.

\section{Organotypic 3D Skin Reconstructs}

Organotypic 3D skin reconstructs were generated as previously described ${ }^{30}$. In each insert, $6.4 \times 10^{4}$ fibroblasts were plated on top of the acellular layer (BD \#355467 and Falcon \#353092) and incubated for $45 \mathrm{~min}$ at $37^{\circ} \mathrm{C}$ in a $\% \mathrm{CO} 2$ tissue culture incubator. DMEM containing $10 \%$ FBS was added to each well of the tissue culture trays and incubated for 4 days. Reconstructs were then incubated for $1 \mathrm{~h}$ at $37^{\circ} \mathrm{C}$ in HBSS containing $1 \%$ dialyzed FBS (wash media). Washing media was removed and replaced with reconstruct media I. Keratinocytes $\left(4.17 \times 10^{5}\right)$ and melanoma cells $\left(8.3 \times 10^{4}\right)$ were added to the inside of each insert. Media was changed every other day until day 18 when reconstructs were harvested, fixed in 10\% formalin, paraffin embedded, sectioned and stained. Quantification of the invasion was performed using ImageJ software (available at http://imagej.nih.gov/ij/; developed by Wayne Rasband, National Institutes of Health, Bethesda, MD)

\section{D Spheroid Assays}

Tissue culture-treated 96-well plates were coated with $50 \mu \mathrm{l}$ 1.5\% Difco Agar Noble (Becton Dickinson). Melanoma cells were seeded at $5 \times 10^{3}$ cells / well and allowed to form spheroids over $72 \mathrm{~h}$. Spheroids were harvested and embedded as previously described using collagen type I (GIBCO, \#A1048301). For spheroids incubated with fibroblast conditioned media, fibroblasts were seeded onto $75 \mathrm{~cm}^{2}$ flasks at $7-9 \times 10^{5} /$ flask depending on growth rate. Sixteen hours later, media was replaced and incubated for $72 \mathrm{~h}$. Media from young fibroblasts was combined and media from aged fibroblasts was combined. This conditioned media was added to the top of the collagen plug containing the spheroids. Quantitation of invasive surface area was performed using NIS Elements Advanced Research software.

\section{Live/dead staining}

Spheroids were generated and embedded as described above. Spheroids were stained using LIVE/DEAD® Viability/Cytotoxicity Kit (L3224, Invitrogen). Briefly, spheroids were washed with PBS and stained with calcein AM/Ethidium homodimer-1. The dyes were diluted in PBS and $300 \mu \mathrm{l}$ of the solution was added on the spheroid wells for 1 hour at $37^{\circ} \mathrm{C}$. The spheroids were washed in PBS and imaged using Nikon TE2000 Inverted Microscope. 
Quantitation of fluorescence intensity was performed using NIS Elements Advanced Research software.

\section{Boyden Chamber Invasion assays}

Matrigel (BD Biosciences, \#354234) was diluted in PBS (1:3000 dilution). 150 $\mu$ of this mixture was pipetted into each insert of the invasion assay plate (Corning, \#3422). The plate was incubated at $37^{\circ} \mathrm{C}$ for 2 hours and then dried at room temperature overnight under sterile conditions. Melanoma cells were pre-treated for 48 hours in 6 well plates. After 48 hours, the cells were harvested and $1.5 \times 10^{5}$ cells were added to each transwell. High concentration serum media (RPMI with $20 \%$ FCS, Tumor growth media with $10 \%$ FCS) was added to the outside (bottom) of the well. The plates were incubated at $37^{\circ} \mathrm{C}$ until cells had migrated to the bottom of the well. The migrated cells were fixed in $95 \%$ ice-cold methanol and stained with crystal violet $(0.5 \%)$ for 10 minutes. The stain was washed and the wells were left to dry. Cells were imaged and quantified using ImageJ software.

\section{Cellular Proliferation assays}

In a 24 well plate, 5000 cells in triplicate were plated per day of measurement. Every 2-3 days, cells were counted using hemocytometer and the total cell number in the well was recorded and plotted on Graphpad/Prism.

\section{Immunofluorescence (IF)}

Cells were seeded onto glass cover slips at $1-4 \times 10^{4}$ cells /well, and incubated overnight. After treatment, cells were fixed using $95 \%$ methanol. Primary antibodies were diluted as stated above in blocking buffer and incubated overnight at $4{ }^{\circ} \mathrm{C}$. Cells were washed in PBS and incubated with the appropriate secondary antibody (1:2000, Invitrogen) for $1 \mathrm{~h}$ at room temperature. Cells were then washed in PBS and mounted in Prolong Gold anti-fade reagent containing DAPI (Invitrogen). Images were captured on a Leica TCS SP5 II scanning laser confocal system.

\section{Immunohistochemistry (IHC)}

All antibodies are described in the Supplementary Information. Patient samples were collected under IRB exemption approval for protocol \#EX21205258-1. Paraffin embedded sections were rehydrated through a xylene and alcohol series, rinsed in $\mathrm{H}_{2} \mathrm{O}$ and washed in PBS. Antigen retrieval was performed using target retrieval buffer (Vector Labs, Burlingame, CA) and steamed for $20 \mathrm{~min}$. Samples were then blocked in a peroxidase blocking buffer (Thermo Scientific) for $15 \mathrm{~min}$, followed by Protein block (Thermo Scientific) for $5 \mathrm{~min}$, and incubated in appropriate primary antibody diluted in antibody diluent (S0809, Dako) at $4^{\circ} \mathrm{C}$ overnight in a humidified chamber. For mouse samples to be incubated with anti-mouse antibody, samples were blocked for 1 hour in Mouse on Mouse (M.O.M. ${ }^{\mathrm{TM}}$ ) Blocking Reagent (MKB-2213, Vector Labs). Following washing in PBS, samples were incubated in biotinylated anti-rabbit or polyvalent secondary antibody (Thermo Scientific) followed by streptavidin-HRP solution at room temperature for $20 \mathrm{~min}$. Samples were then washed in PBS and incubated in 3-Amino-9-Ethyl-1-Carboazole (AEC) 
chromogen and counter stained with Mayers hematoxylin for $1 \mathrm{~min}$, rinsed in cold $\mathrm{H}_{2} \mathrm{O}$, and mounted in Aquamount.

\section{Western Blotting}

Total protein lysate (50-65 $\mu \mathrm{g}$ ) was run on a 4-12\% NuPAGE Bis Tris gel (Invitrogen). Proteins were then transferred onto PVDF membrane using an iBlot system, and blocked in $5 \%$ milk/TBST for 1 hour. All primary antibodies were diluted in 5\% milk/TBST and incubated over night at $4^{\circ} \mathrm{C}$. The membranes were washed in TBST and probed with the corresponding HRP-conjugated secondary antibody $(0.2-0.02 \mu \mathrm{g} / \mathrm{ml}$ of anti-mouse, streptavidin, or anti-rabbit). Proteins were visualized using, ECL prime (Amersham, Uppsala, Sweden), or Luminata Crescendo (Millipore, Billerica, MA).

\section{Lentiviral infection}

All clones used are described in the Supplementary Information. All shRNA was obtained from the TRC shRNA library available through the Molecular Screening Facility at The Wistar Institute. Lentiviral production was carried out as described in the protocol developed by the TRC library (Broad Institute). Briefly, 293T cells were co-transfected with shRNA vector and lentiviral packaging plasmids (pCMV-dR8.74psPAX2, pMD2.G). The supernatant containing virus was harvested at 36 and 60 hours, combined and filtered through a $0.45 \mu \mathrm{m}$ filter. For transduction, the cells were layered overnight with lentivirus containing $8 \mu \mathrm{g} / \mathrm{ml}$ polybrene. The cells were allowed to recover for 24 hours and then selected using $1 \mu \mathrm{g} / \mathrm{ml}$ puromycin.

\section{MTS Assays}

Cells $\left(1.5 \times 10^{3} /\right.$ well $)$ were plated in a 96 well plate and treated with PLX4720. After $48 \mathrm{~h}$, cells were incubated with MTS dye (20 $\mu \mathrm{l} /$ well) for $2 \mathrm{~h}$. Absorbance was determined at 490 nm using an EL800 microplate reader (BioTek, Winooski, VT). The percent cell proliferation was calculated by converting the experimental absorbance to percentage of control and plotted vs drug concentration. The values were then analyzed using a nonlinear dose-response analysis in GraphPad Prism.

\section{Illumina oligonucleotide microarray}

Transcriptional profiling was determined using Illumina Sentrix BeadChips. Total RNA was used to generate biotin-labeled cRNA using the Illumina TotalPrep RNA Amplification Kit. In short, $0.5 \mu \mathrm{g}$ of total RNA was first converted into single-stranded cDNA with reverse transcriptase using an oligo-dT primer containing the T7 RNA polymerase promoter site and then copied to produce double-stranded cDNA molecules. The double stranded cDNA was cleaned and concentrated with the supplied columns and used in an overnight in-vitro transcription reaction where single-stranded RNA (cRNA) was generated incorporating biotin-16-UTP. A total of $0.75 \mu \mathrm{g}$ of biotin-labeled cRNA was hybridized at $58^{\circ} \mathrm{C}$ for $16 \mathrm{~h}$ to Illumina's Sentrix Human HT-12 v3 Expression BeadChips (Illumina, San Diego, CA). Each BeadChip has around 48,000 transcripts with approximately 15 -fold redundancy. The arrays were washed, blocked and the labeled cRNA was detected by staining with streptavidin-Cy3. Hybridized arrays were scanned using an Illumina BeadStation 500× Genetic Analysis 
Systems scanner and the image data extracted using the Illumina GenomeStudio software, version 1.1.1). Data are available in the GEO database (accession \# GSE57445).

\section{Microarray analysis}

Microarray expression data was quantile normalized and probes that showed low expression levels (detection p-value>0.05) across all samples were removed from the analysis. Expression values for each cell line were tested separately in multiple linear regression model with fibroblast age and experiment batches as predictor variables. Matlab v.8.0 "regress" function was used to calculated p-values for each probe for association with fibroblast age. False Discovery Rate (FDR) was estimated using Benjamini-Hochberg procedure and only probes that showed FDR $<5 \%$ in all 3 cell lines were considered significant. Heatmap was plotted using average expression values for 3 groups of age (young, middle and aged) normalized to aged group (100\%).

\section{In vivo Tail Vein Metastases Assay}

All animal experiments were approved by the Institutional Animal Care and Use Committee (IACUC) (IACUC \#112503Y_0) and were performed in an Association for the Assessment and Accreditation of Laboratory Animal Care (AAALAC) accredited facility. Male C57BL6 mice 6-8 weeks (young) and 52 weeks of age (aged) were purchased from Taconic. YUMM $1.7\left(1 \times 10^{6}\right.$ cells $\left./ 100 \mu \mathrm{PBS}\right)$ or B16F10 $\left(2.5 \times 10^{5} / 100 \mu \mathrm{PBS}\right)$ were injected into the tail vein of C57BL6 mice. Alternatively, Yumm1.7 cells were overexpressed with mCherry plasmid (pLU-EF1-MCS-mcherry) using lentivirus. The cells were sorted for mcherry and $1 \times 10^{6}$ cells $/ 100 \mu \mathrm{l}$ PBS were injected into tail vein of young C57BL6 mice. After 4 weeks, the mice were euthanized, lungs were harvested and metastases counted. Lungs were fixed in paraffin and stained with H\&E. Alternatively, lungs were harvested and imaged for presence of metastatic melanoma cells using Perkin-Elmer IVIS 200 whole body imager. For experiments requiring recombinant sFRP2, mouse recombinant sFRP2 (1169-FR-025/CF,

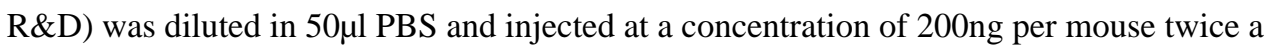
week. The levels of sFRP2 were monitored by submandibular blood withdrawal every two weeks.

\section{In vivo PLX4720 assay}

All animal experiments were approved by the Institutional Animal Care and Use Committee (IACUC) (IACUC \#112503X_0) and were performed in an Association for the Assessment and Accreditation of Laboratory Animal Care (AAALAC) accredited facility. YUMM1.7 $\left(2.5 \times 10^{5}\right.$ cells $)$ were suspended in Matrigel $(500 \mu \mathrm{g} / \mathrm{ml})$ and injected subcutaneously into young ( 6 week) and aged (52 week) C57/BL6 mice (Taconic). When resulting tumors reached $200 \mathrm{~mm}^{3}$, mice were fed either AIN-76A chow or AIN-76A chow containing 417 $\mathrm{mg} / \mathrm{kg}$ PLX4720. Tumor sizes were measured every 3-4 days using digital calipers, and tumor volumes were calculated using the following formula: volume $=0.5 \times$ (length $\times$ width $^{2}$ ). Time-to-event (survival) was determined by a 5 -fold increase in baseline volume $(\sim 1000 \mathrm{~mm} 3)$ and was limited by the development of skin necrosis. Upon the occurrence of necrosis, mice were euthanized. For subsequent experiments involving sFRP2 manipulation, Yumm1.7 cells overexpressing mCherry were used. $1 \times 10^{6}$ cells were suspended in PBS and subcutaneously injected into either 6-week-old or 52-week-old male C57/BL6 mice 
(Taconic). For treatment with recombinant sFRP2, the mice were injected with recombinant protein $(200 \mathrm{ng} / \mathrm{ml})$ through the tail vein every 2 days as described above. For the experiments performed with sFRP2 blocking antibody (clone 80.8.6, \#MABC539, EMD Millipore), the mice were treated with $1 \mathrm{mg} / \mathrm{kg}$ antibody (either sFRP2 or isotype control, Biolegend, \#400264) once per week via tail vein injections.

\section{Comet Assay}

WM35 and FS5 melanoma cells were seeded in 12 well plates and treated with conditioned media for 48 hours. The cells were harvested in ice cold PBS. The comet assay was performed using CometSlides (Trevigen). Briefly, $75 \mu \mathrm{l}$ of a $2 \times 10^{5}$ cell suspension was mixed with $500 \mu 11 \%$ low melting point agarose. $50 \mu \mathrm{l}$ of cell/agarose mixture was dropped into the wells and allowed to solidify. Slides were incubated in lysis buffer $(1.2 \mathrm{M} \mathrm{NaCl}$, 100nM EDTA, 0.1\% Sarkosyl, pH10.0) for $1 \mathrm{~h}$ at $4^{\circ} \mathrm{C}$. Slides were then electrophoresed at $25 \mathrm{~V}$ for $12 \mathrm{~min}$ in alkaline buffer $(0.03 \mathrm{M} \mathrm{NaOH}, 2 \mathrm{mM}$ EDTA, pH8.0). After fixation in $70 \%$ ethanol, comets were visualized by staining with SYBR Green (Fisher). The extent of DNA damage was measured as the artificial Olive Moment using Cometscore software downloaded from http://www.tritekcorp.com.

\section{ROS Activation Assay}

5000 melanoma cells were seeded in a 96 well plate in triplicate. The cells were then treated with conditioned media from young and aged fibroblasts as well as DMEM as control. Hydrogen peroxide was added at $1 \mathrm{mM}$ as control. After 72 hours, reactive oxygen species were measured using Cell Meter ${ }^{\mathrm{TM}}$ Fluorimetric Intracellular Total ROS Activity Assay Kit (\#22901, AAT Bioquest, CA) according to the manufacturer's protocol. The plates were measured using the PerkinElmer EnVision Xcite Multilabel plate reader using the filters for Ex/Em=520/605 nm. Alternatively, samples were imaged using PerkinElmer Operetta and the fluorescent signal was quantified using Harmony 3.0 software. The cell number was determined by Hoescht staining (Hoechst 33342, Invitrogen) and used to normalize the total fluorescence obtained from ROS staining.

\section{Topflash assay}

Topflash vectors were obtained from Addgene (M51 Super $8 \times$ FOPFlash/TOPFlash mutant, \#12457; M50 Super 8× TOPFlash, 12456). WM35 cells were plated to achieve 70\% confluency in 6-well plates. Cells were co-transfected with pTK-RLuc (Green Renilla Luciferase) along with either Topflash or Fopflash vectors. After 5 hours of transfection, cells were treated as required. After 48h, cells were harvested and luciferase activity was measured using Dual-Luciferase ${ }^{\circledR}$ Reporter (DLR ${ }^{\mathrm{TM}}$ ) Assay System (Promega, E1910). The firefly luciferase signal from each well was normalized to its Renilla Luciferase signal. Topflash/fopflash signal was determined from each treatment and graphed using Graphpad/ Prism. 


\section{Treatment with $\mathrm{N}$-acetyl cysteine}

N-Acetyl-L-cysteine (NAC) was obtained from Sigma (A9165) and dissolved in sterile $\mathrm{dH}_{2} \mathrm{O}$ (stock $1 \mathrm{M}$ ). Cells were treated for $48 \mathrm{~h}$ and analyzed. After optimization, $10 \mathrm{mM}$ final concentration was used for subsequent experiments.

\section{Cell Fractionation}

Melanoma cells were seeded into T25 flasks and incubated for 72 hours with $6.5 \mathrm{ml}$ conditioned fibroblast media prepared as described above. Cells were then washed in PBS, harvested with TrypLE Express and fractionated using the cellular fractionation kit (NEPER, Fisher) as per manufacturer's protocol. Cell lysates were then separated on an SDSPAGE gel and visualized using standard Western blotting procedures.

\section{ELISA}

Nunc $^{\mathrm{TM}}$ MaxiSorp ${ }^{\mathrm{TM}}$ ELISA plates (ebiosciences, CA) were coated with $50 \mu \mathrm{l}$ of $3 \mu \mathrm{g} / \mathrm{ml}$ sFRP2 (\#ab137560, abcam) overnight at $4^{\circ} \mathrm{C}$. Plates were washed in PBS containing $0.1 \%$ Tween and blocked in ELISA diluent (00-4202-56, ebiosciences, CA) for 2 hours. Serum was diluted 1:100 before addition to the plates and incubated overnight at $4^{\circ} \mathrm{C}$. Next day, the plates were washed in PBS containing 0.1\% Tween20 and incubated with detection antibody (MAB6838, R\&D systems, MN) for $1 \mathrm{~h}$ at room temperature. Plates were washed and incubated with secondary antibody for $1 \mathrm{~h}$. After washing, 100 $\mu \mathrm{l} \mathrm{TMB} \mathrm{(00-4201-56,}$ ebiosciences, CA) was added to the plates and incubated for 15 minutes. The reaction was stopped using $50 \mu \mathrm{l}$ of $2 \mathrm{~N} \mathrm{H}_{2} \mathrm{SO}_{4}$ and absorbance was measured at $450 \mathrm{~nm}$.

\section{$\beta$-galactosidase staining}

Fibroblasts were plated into 12-well dishes, incubated for $48 \mathrm{~h}$, washed with PBS and fixed in $2 \%$ formaldehyde $/ 0.2 \%$ glutaraldehyde. Cells were then incubated in staining solution (150mM NaCl, Sigma, St. Louis, MO), $2 \mathrm{mM} \mathrm{MgCl}_{2}$ (Sigma), $5 \mathrm{mM} \mathrm{K}_{3} \mathrm{Fe}(\mathrm{CN})_{6}$ (Millipore), $5 \mathrm{mM} \mathrm{K}_{4} \mathrm{Fe}(\mathrm{CN})_{6}$ (Millipore), $40 \mathrm{mM} \mathrm{Na}_{2} \mathrm{PO}_{4}$ (Sigma) pH5.5, 20mg/ml X-gal (Applichem, Darmstadt, Germany) at $37^{\circ} \mathrm{C}$ overnight. Stain was removed and cells were stored in $70 \%$ glycerol before being imaged.

\section{Quantitative PCR}

All primers are listed in the Supplementary Information. Mouse tissue was snap frozen in liquid nitrogen immediately after harvesting. 10mg of the lung tissue was homogenized and RNA was extracted using Trizol (Invitrogen) and RNeasy Mini kit (Qiagen) as described previously. 1 $\mu \mathrm{g}$ RNA was used to prepare cDNA using iscript DNA synthesis kit (\#1708891, Bio-Rad, CA). cDNA was diluted 1:5 before use for further reactions. Each 20 $\mu$ l well reaction comprised of 10 $\mu$ l Power SYBR Green Master mix (4367659, Invitrogen), $1 \mu \mathrm{l}$ primer mix (Final concentration $0.5 \mu \mathrm{M}$ ) and $1 \mu \mathrm{cDNA}$. Standard curves were generated for all primers and each set of primers were normalized to 18 Primer pair.

\section{Statistical Analysis}

For in vitro studies, a Student's t-test or Wilcoxon rank-sum test (Mann Whitney) was performed for two-group comparison. Estimate of variance was performed and parameters 
for the t-test were adjusted accordingly using Welch's correction. ANOVA or Kruskal-Wallis test with post-hoc Bonferroni's or Holm-Sidak's adjusted p-values was used for multiple comparisons. For dose response analysis, Spearman's correlation was calculated. For in vivo studies, the indicated sample size for each experiment was designed to have $80 \%$ power at a two-sided alpha of 0.05 to detect a difference of large effect size about 1.25 between two groups on a continuous measurement. The fold change in tumor volume at each time point after treatment relative to baseline was calculated and then the fold change in treatment group relative to age matched control group was used with a mixed-effect model to evaluate the treatment effect between age groups. Stata 12.0 (StatCorp LP, College Station, TX, USA) was used for data analysis for in vivo studies and human samples. For other experiments, Graphpad/Prism6 was used for plotting graphs and statistical analysis. Significance was designated as follows: *, $<<0.05 ; * *, p<0.01 ; * * *, p<0.001$. Extended statistical analyses for patient data are provided in the Supplementary Information.

\section{Extended Data}
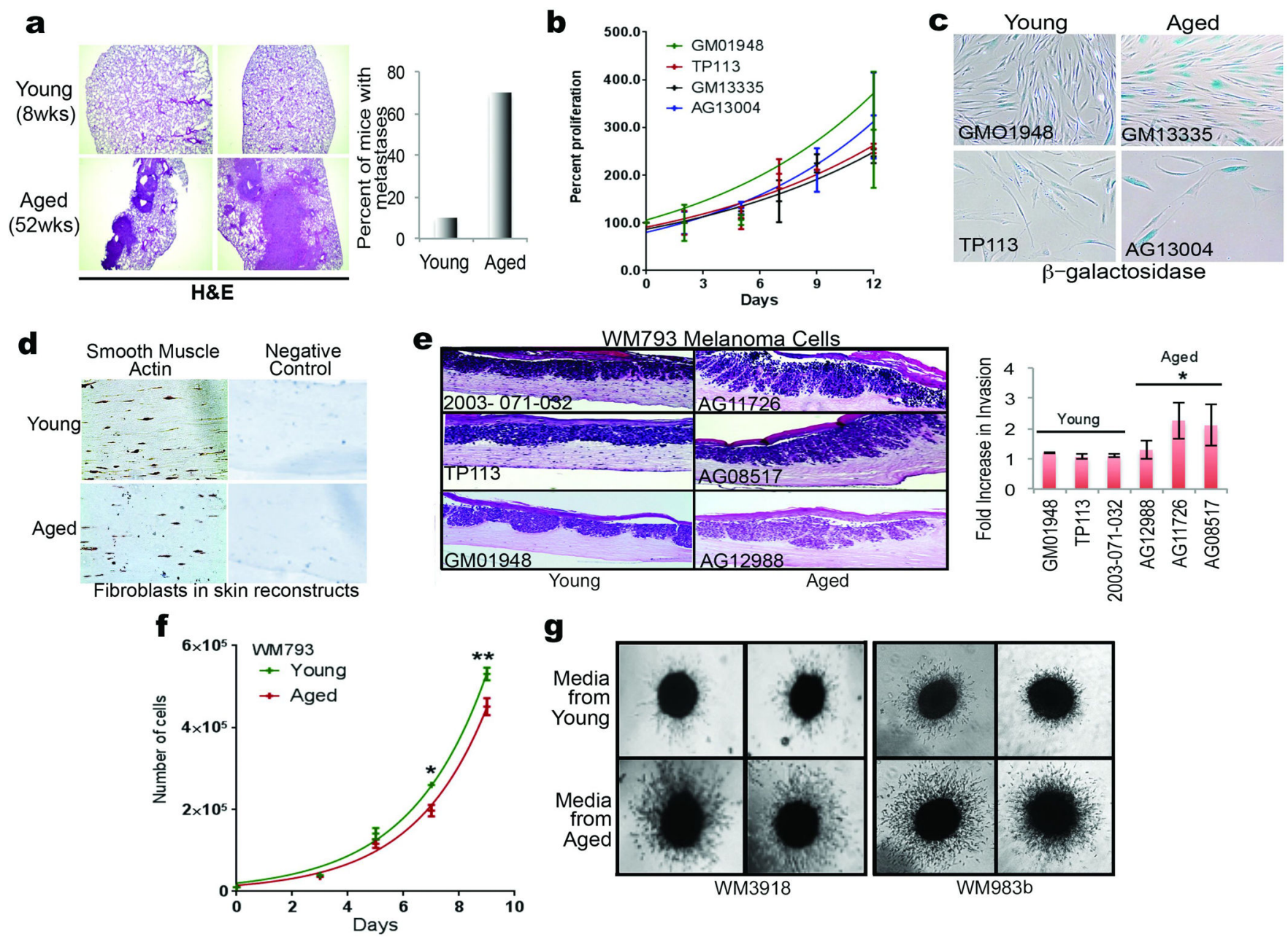

g

Extended Data 1. Characterization of young and aged fibroblasts 
$1 \times 10^{6}$ Yumm1.7 cells were injected into the tail vein of young ( 8 weeks, $\mathrm{n}=10$ mice) and aged (52 weeks, $\mathrm{n}=10$ mice) mice, and 3 weeks later, lungs were assessed for metastatic colonies. Samples were analyzed by H\&E staining. Number of mice with metastatic colonies in the lungs was quantified in graph (a). Proliferation rate of aged and young fibroblasts was measured by simple cell counts over a period of 12 days. Analysis of variance (ANOVA) is insignificant ( $\mathrm{p}=0.234$ ) (b). Young and aged fibroblasts were assessed for basal $\beta$-galactosidase activity after 5 passages in culture. Representative images from 2 cell lines are shown for young and aged fibroblasts, magnification $100 \times(\mathbf{c})$. Staining of fibroblasts in skin reconstructs with a-SMA-1 to assess persistence of fibroblasts in cell culture. Representative images, magnification 150× (d). WM793 melanoma cells were grown in organotypic 3D skin reconstructs built with 3 different fibroblast cell lines derived from healthy young (25-35) and healthy aged (55-65) individuals. Representative images, magnification 150x. Invasion was quantified using NIS Element software. Analysis of variance (ANOVA) was performed ( $\mathrm{p}=0.007)$. Holm-Sidak multiple comparisons comparing each young cell line with each aged cell line indicated $\mathrm{p}<0.05$ (e). WM793 melanoma cells exposed to conditioned media from young and aged fibroblasts were assessed for proliferation using simple cell counts. Repeated measures analysis of variance (ANOVA) was calculated between samples ( $\mathrm{p}=0.006$ ). Bonferroni's multiple comparisons test on day 7 and 9 was performed to obtain adjusted $\mathrm{p}$ value [day 7 ( $\mathrm{p}=0.047)$, day 9 ( $\mathrm{p}=0.0004)$ ] (f). Multiple melanoma cells were allowed to form spheroids followed by treatment with conditioned media from aged or young fibroblasts for $48 \mathrm{~h}$. The spheroids were then examined for their ability to invade in a collagen matrix (g). Data are represented as mean \pm s.d. for each graph $(\mathbf{b}, \mathbf{e}, \mathbf{f})$. 
a

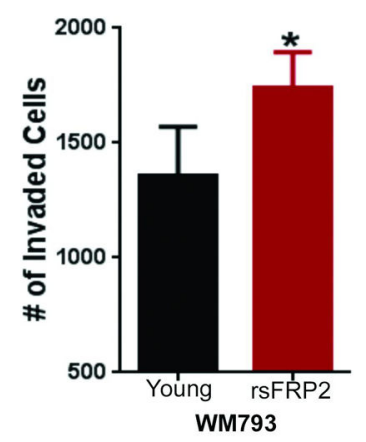

b

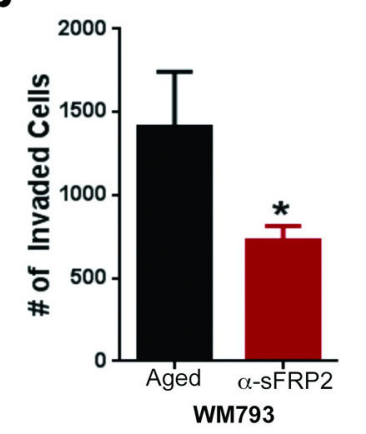

C

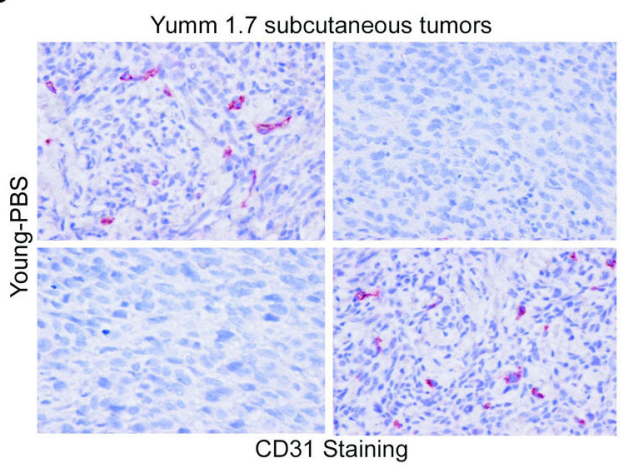

e

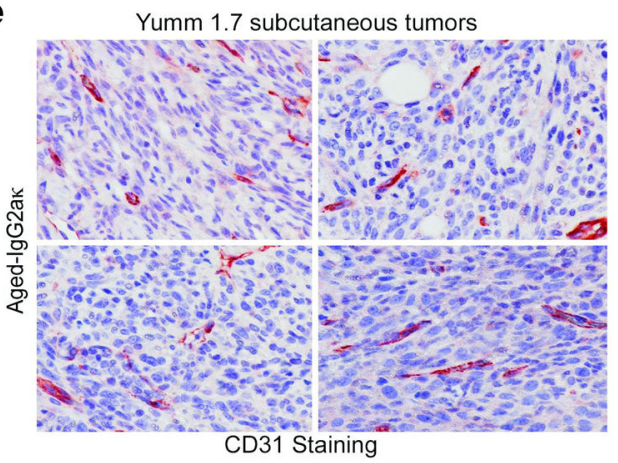

d

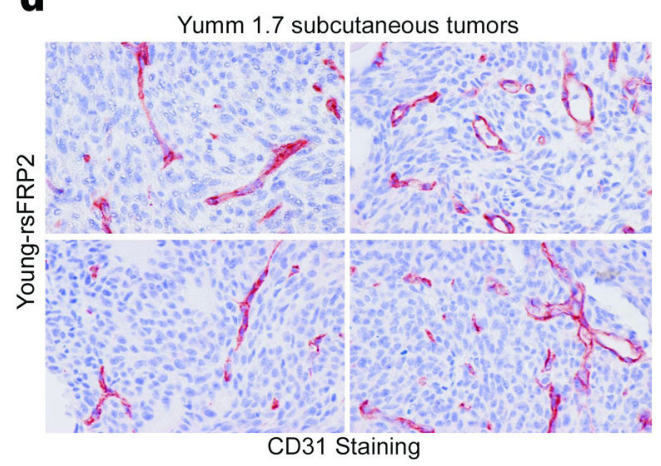

$\mathbf{f}$

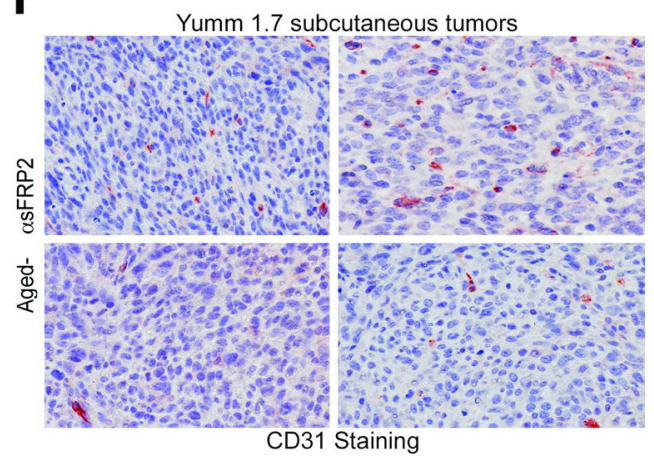

Extended Data 2. SFRP2 promotes invasion and angiogenesis

Conditioned media from young fibroblasts treated with either control (PBS), or rsFRP2

(200ng/ml) was used to pre-treat WM793 melanoma cells for 48h. Invasion was assayed using a Boyden Chamber assay over 24-72h. Two-tailed unpaired t test was performed $(\mathrm{p}=0.033)$ (a). Conditioned media from aged fibroblasts treated with sFRP2 blocking antibody $(15 \mu \mathrm{g} / \mathrm{ml})$ for $72 \mathrm{~h}$ was used to pre-treat WM793 melanoma cells for $48 \mathrm{~h}$. The invasion of melanoma cells was assessed in a Boyden Chamber assay for 24-72h. Twotailed unpaired $\mathrm{t}$ test was performed ( $\mathrm{p}=0.035)$ (b). Young mice ( 8 weeks, 10 /group) were injected subcutaneously with Yumm1.7 cells. After palpable tumor appeared, mice were treated with rsFRP2 $(200 \mathrm{ng} / \mathrm{mL})$ for 30 days and examined for angiogenesis using CD31 staining. Representative images, magnification $400 \times(\mathbf{c}, \mathbf{d})$. Aged mice ( 52 weeks, $n=5$ / group) were injected subcutaneously with Yumm1.7 cells and treated with either control IgG2ak or sFRP2 blocking antibody $(1 \mathrm{mg} / \mathrm{kg})$ for 3 weeks. Tumors were examined for angiogenesis by CD31 staining. Representative images, magnification $400 \times(\mathbf{e}, \mathbf{f})$. Data are represented as mean \pm s.d. for each graph $(\mathbf{a}, \mathbf{b})$. 
a

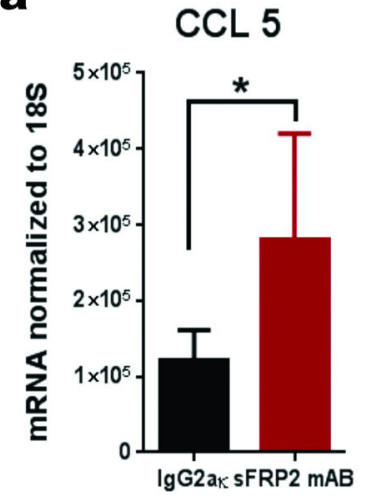

e

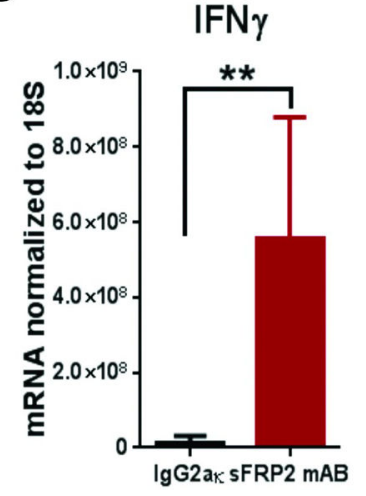

b

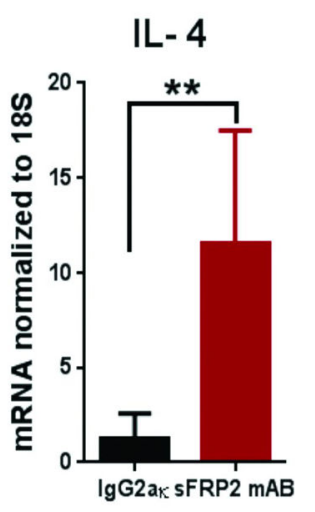

f

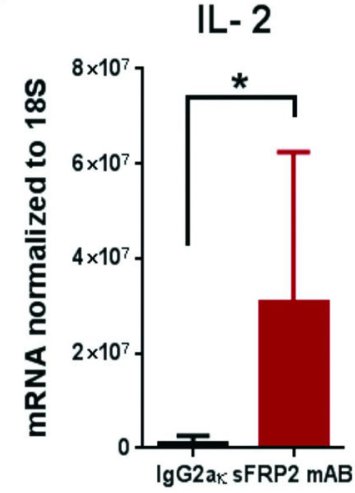

C IL-10

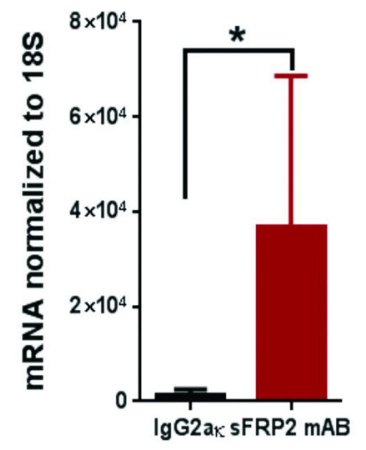

$\mathbf{g}$

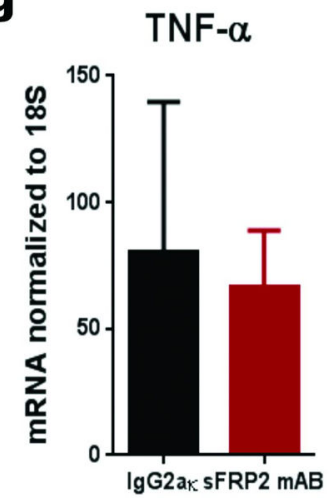

d

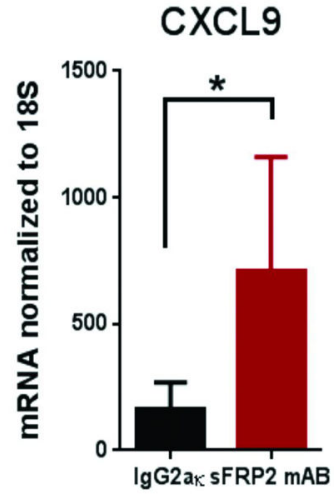

h

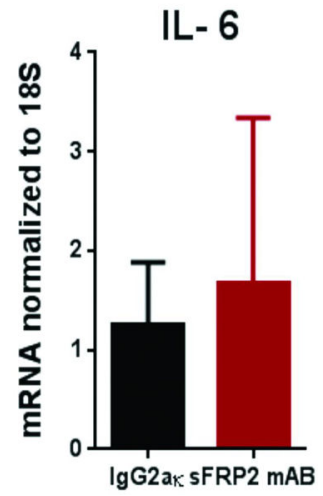

Extended Data 3. Treatment of aged tumor bearing mice with an a-sfrp2 antibody results in a lethal inflammation

Cytokine analysis of lungs in aged tumor-bearing mice ( 52 weeks, $n=5$ /group) treated with $\operatorname{IgG} 2 \mathrm{a} \kappa$ or a-sFRP2 antibody ( $1 \mathrm{mg} / \mathrm{kg}$, once weekly for 3 weeks). RT-PCR demonstrates a difference in the lungs of mice treated with IgG2ak or a-sFRP2 in cytokines CCL5 (a), IL4 (b), IL10 (c), CXCL9 (d), IFN $\gamma$ (e), and IL2 (f). Early response inflammatory genes TNFa (g) and IL6 (h) were no longer significantly altered. Estimate of variance was performed for all genes. For all cytokines, two-tailed unpaired t test was performed $*=p<0.05, * *=p<0.02$.

Data are represented as mean \pm s.d. for each graph. 
a

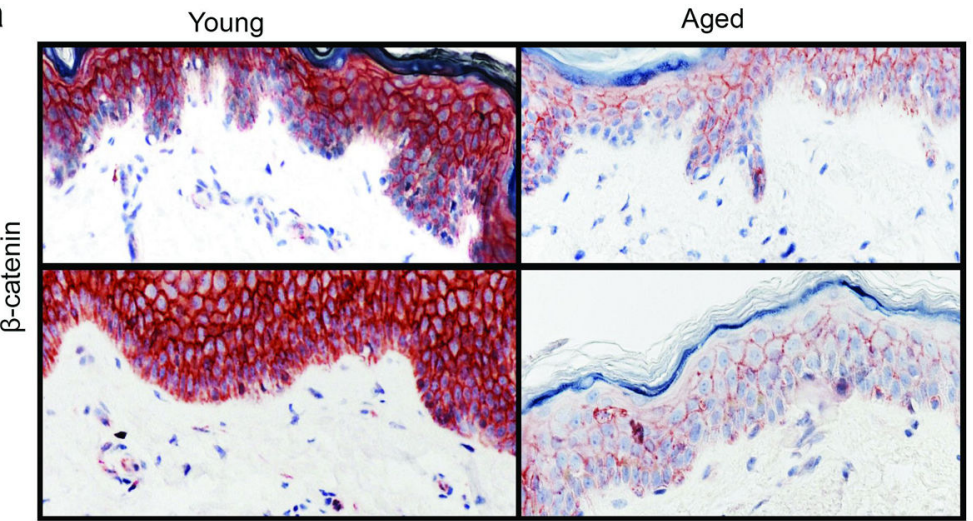

Human Skin (no tumor; $n=19$ ) b

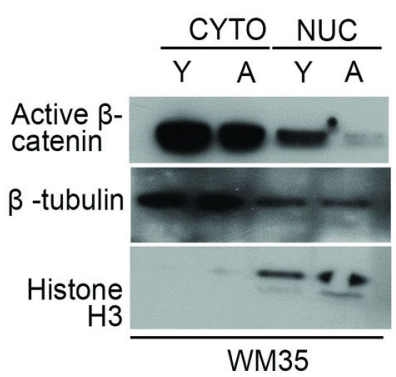

C

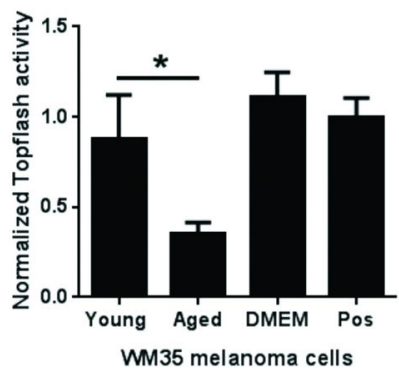

d

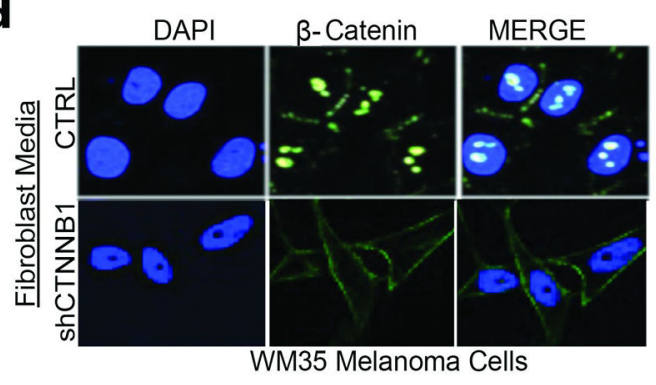

Extended Data 4. $\beta$-catenin loss in the aged microenvironment

$\beta$-catenin expression in normal human skin from young and aged donors, with a focus on the fibroblast population (zoom) (a). $\beta$-catenin nuclear translocation in melanoma cells treated with conditioned media from aged as compared to young fibroblasts as measured by Western analysis (b) and a TOPFLASH assay. Two-tailed unpaired $t$ test was performed to indicate statistical significance between treatment with young and aged media $(\mathrm{p}=0.023)$. Data are represented as mean \pm s.d. (c). Immunofluorescent analysis of $\beta$-catenin in melanoma cells treated with media from young fibroblasts in which the $\beta$-catenin is knocked down (d). 
a
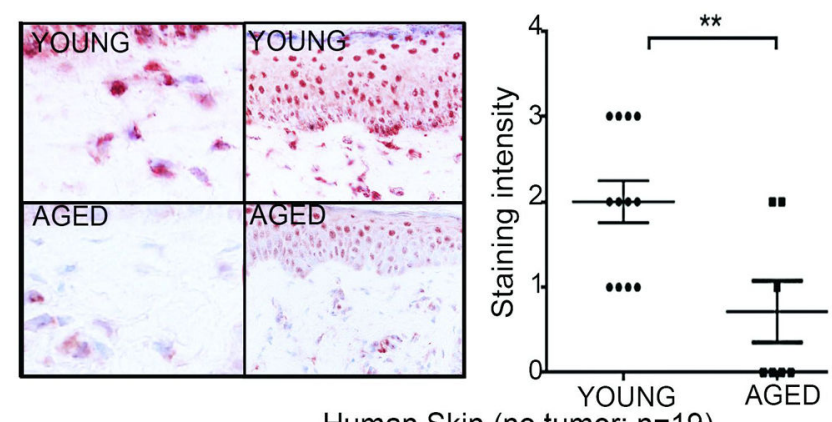

Human Skin (no tumor; $n=19$ ) b

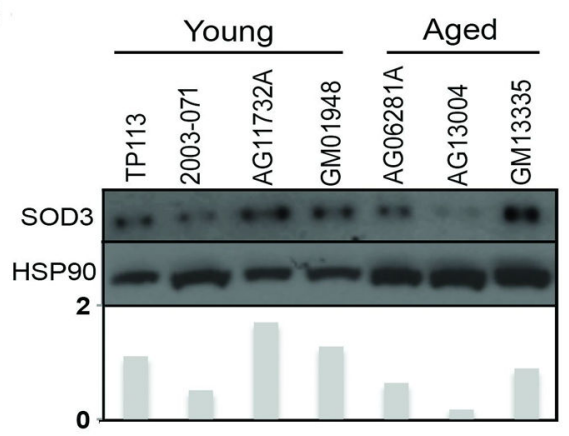

C

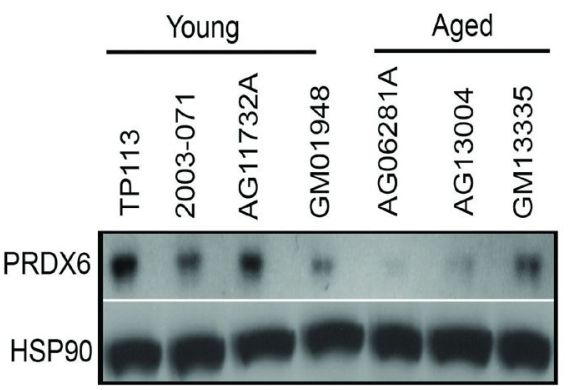

d

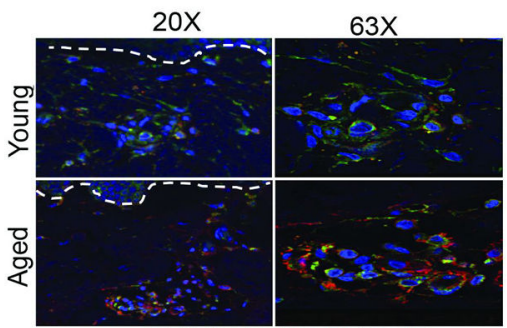

SMA/ DAPI/ 8-OXO-dG e

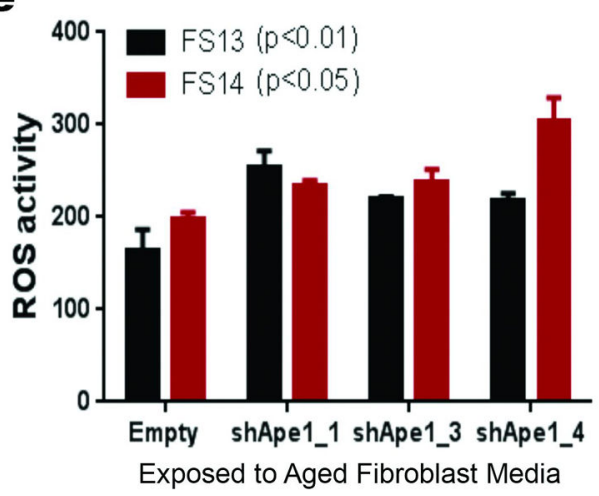

Extended Data 5. Increase in oxidative stress in the aged microenvironment

APE1 expression in normal human skin as measured by immunohistochemistry. Slides were scored for intensity of stain (3-highest, 0-lowest, $<35 \mathrm{yr}, \mathrm{n}=12$; $>55 \mathrm{yr}, \mathrm{n}=7$ ). Representative images, magnification 400× (left) and 200× (right). Unpaired t test using rank sum (Mann Whitney) revealed statistical significance $(\mathrm{p}=0.009)(\mathbf{a})$. Western analysis of SOD3 (b) and PRDX6 (c) levels in conditioned media from young and aged fibroblasts.

Immunofluorescent analysis of 8-oxo-dG in normal young and aged skin stained for oxidative stress marker 8-oxo-dG (red), smooth muscle actin (green) and DAPI (blue) (d). ROS activity in melanoma cells with APE1 knockdown, after exposure to aged media. Analysis of variance was performed for each cell line treatment [FS13 ( $\mathrm{p}=0.0006)$; FS14 $(\mathrm{p}=0.004)]$. For FS13, Two-tailed unpaired t test indicated significance $(\mathrm{p}<0.01)$ each shAPE1 cell line compared to control cells. For FS14, two-tailed unpaired t test indicated significance with $\mathrm{p}<0.05$ each shAPE1 cell line compared to control cells (e). Data are represented as mean \pm s.d. for each graph (a,e). 
a

b
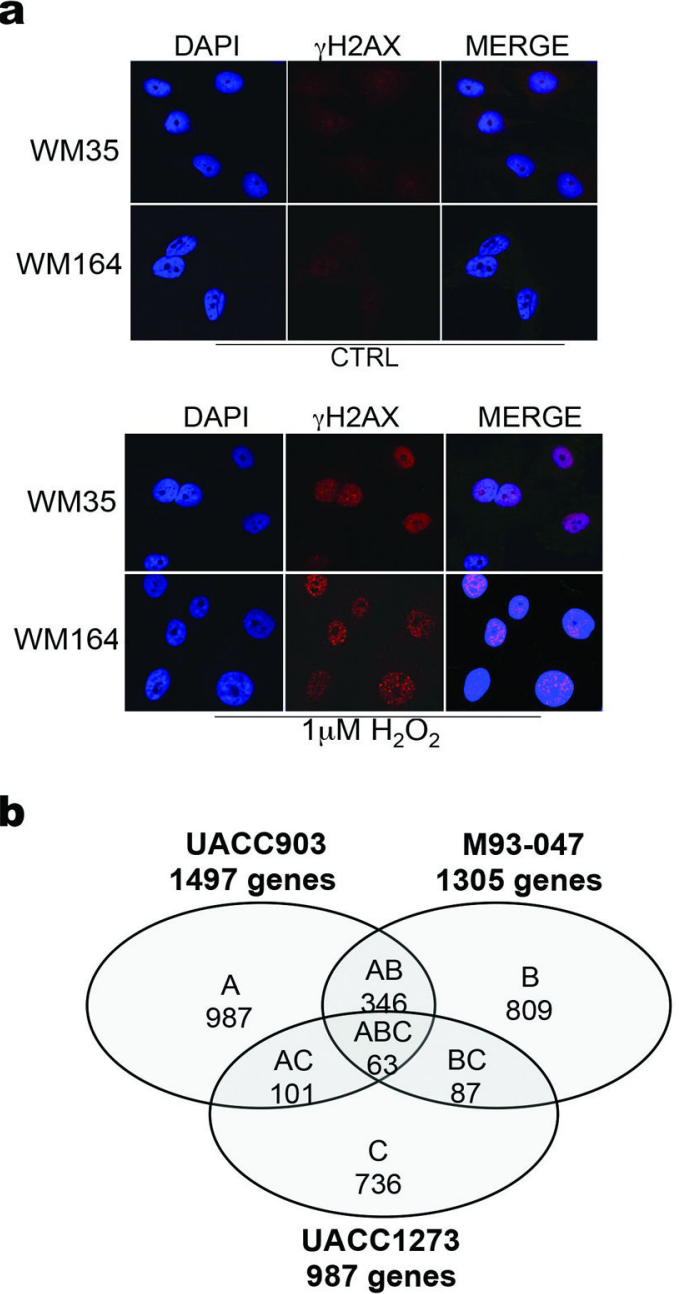

c

\begin{tabular}{|c|c|c|c|c|}
\hline $\begin{array}{l}.903 \\
\mathrm{Y}|\mathrm{M}| \mathrm{A}\end{array}$ & $\begin{array}{l}\text { i.M93 } \\
Y|M| A \mid\end{array}$ & $\begin{array}{l}\text { n.1273 } \\
Y|M| A\end{array}$ & & $\begin{array}{l}\text { Expr sca } \\
\square 100\end{array}$ \\
\hline & & & IL21R : interleukin 21 receptor & \\
\hline & & & PPBP : pro-platelet basic protein & 8 \\
\hline & & & ING1: inhibitor of growth family, member 1 & \\
\hline & & & CCNYL1 : cyclin Y-like 1 & $-50 \%$ \\
\hline
\end{tabular}

PPM1D : protein phosphatase 1D magnesium-dep $\square<50 \%$

AFTPH : aftiphilin

RND3 : Rho family GTPase 3

MIA3 : melanoma inhibitory activity family, member 3

p21 : cyclin-dependent kinase inhibitor $1 \mathrm{~A}$

BTBD7 : BTB (POZ) domain containing 7

LIN37 : lin-37 homolog

RSRC2 : arginine/serine-rich coiled-coil 2

VWCE : von Willebrand factor $\mathbf{C}$ and EGF domains

SEC11C : SEC11 homolog C

MARCH8 : membrane-associated ring finger 8

ASMTL : acetylserotonin O-methyltransferase-like

OSTCL : oligosaccharyltransferase complex subunit-like

BNIP1 : BCL2/adenovirus E1B 19kDa interacting protein 1

TBC1D15 : TBC1 domain family, member 15

UPLP : uroplakin-like protein

SLC25A38 : solute carrier family 25 , member 38

UBFD1 : ubiquitin family domain containing 1

STX5 : syntaxin 5

ZNF133 : zinc finger protein 133

KCMF1 : potassium channel modulatory factor 1

CD2 : CD2 molecule

RMND5A : required for meiotic nuclear division $5 A$

HK2 : hexokinase 2

ATF2 : activating transcription factor 2

BZW1 : basic leucine zipper and W2 domains 1

ZNF259 : zinc finger protein 259

GPR108: G protein-coupled receptor 108

HNRPC : heterogeneous nuclear ribonucleoprotein C

Extended Data 6. Gene expression analysis of melanoma cells exposed to aged fibroblasts reveals increases in DNA damage

$\gamma \mathrm{H} 2 \mathrm{AX}$ expression was analyzed in melanoma cells exposed to $\mathrm{H}_{2} \mathrm{O}_{2}$ using

immunofluorescence (a). Microarray analysis of the gene expression profiles of melanoma cells exposed to young/middle (Y/M) and aged fibroblasts identified 63 genes commonly increased in 3 melanoma cell lines cultured with aged vs young fibroblasts (b). 33 genes involved in DNA damage response were significantly altered due to effects of aging microenvironment in three different melanoma cell lines. Color scale indicates expression levels relative to aged group (c). 
a
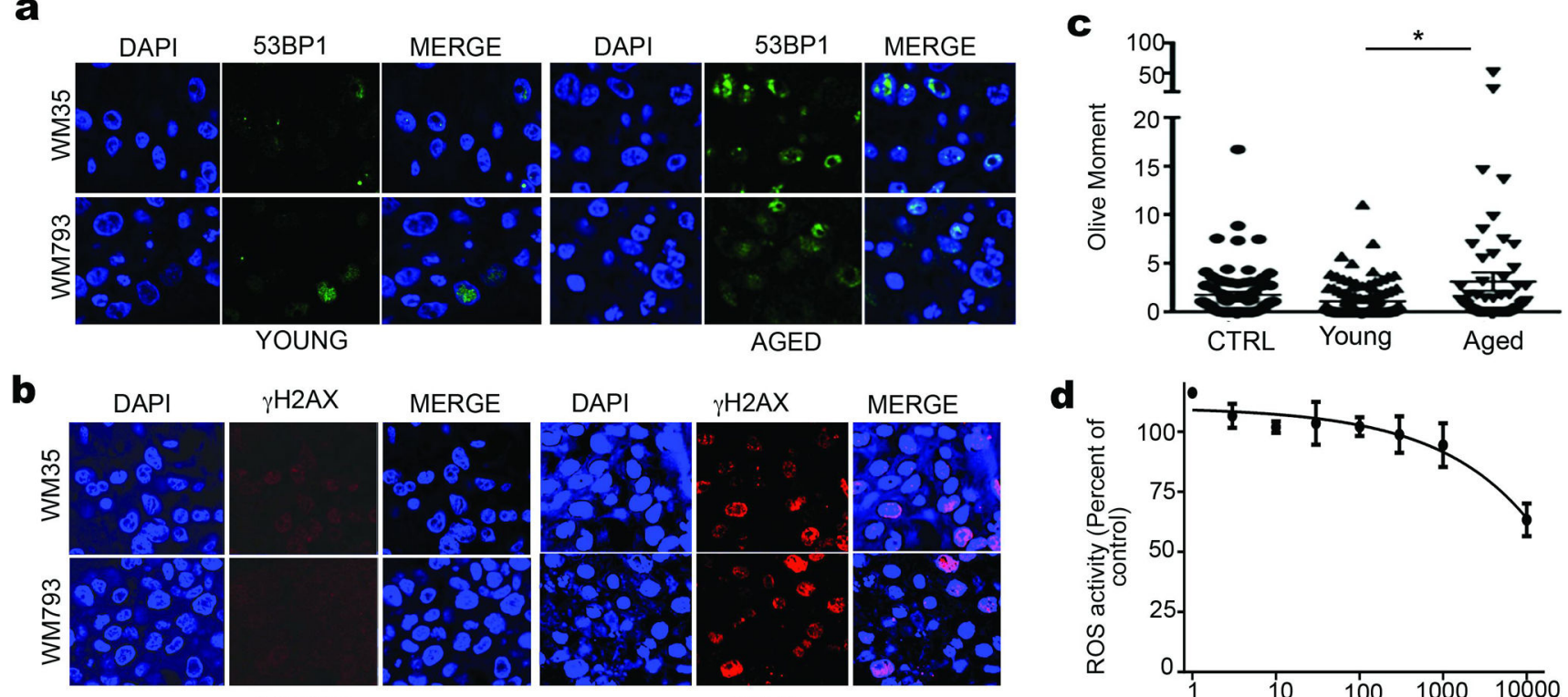

$\gamma \mathrm{H} 2 \mathrm{AX}$

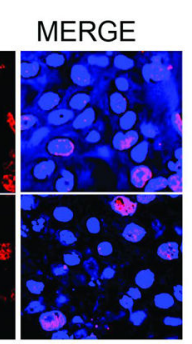

d.

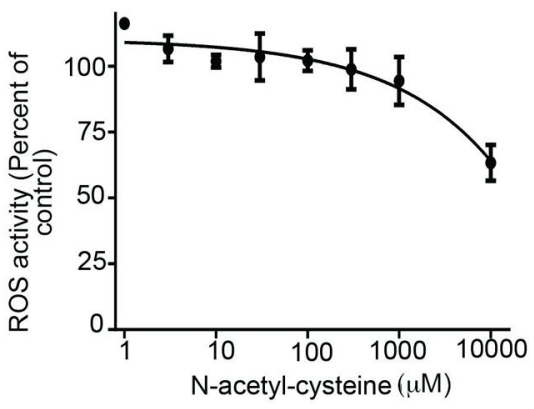

$\mathbf{e}$
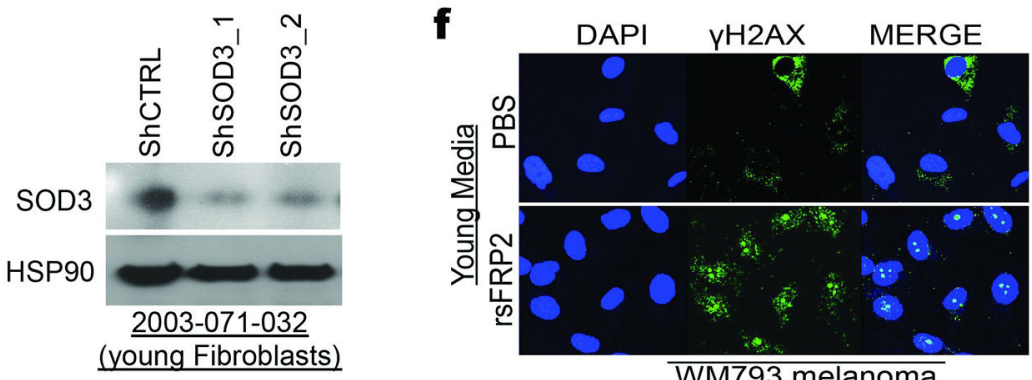

WM793 melanoma

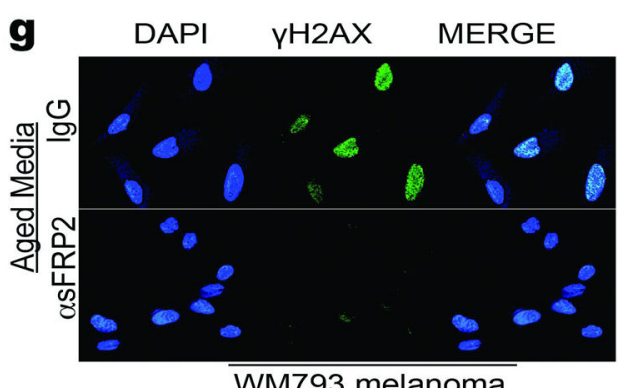

Extended Data 7. DDR response is increased in melanoma cells exposed to aged fibroblasts Skin reconstructs made with young or aged fibroblasts were stained with anti 53BP1 (a) or $\gamma \mathrm{H} 2 \mathrm{AX}$ (b) and analyzed by immunofluorescence. FS5 melanoma cell line treated with conditioned media from young and aged fibroblasts showing DNA damage as measured by a comet assay. Two-tailed unpaired t test with Welch's correction was performed between young and aged treatments $(\mathrm{p}=0.039)$. Data are represented as mean \pm s.e.m. (c). Percent ROS activity remaining after NAC treatment of aged fibroblasts. Spearman's correlation between dose and percent inhibition is significant $[\mathrm{p}=0.043, \mathrm{r}=-0.700]$ Data are represented as mean \pm s.d. (d). Knockdown of SOD3 in young fibroblasts as analyzed by Western blotting (e). Young fibroblasts (2003,071-032 and AG11732) were treated with rsFRP2 (200ng/ml) for $72 \mathrm{~h}$ and this conditioned media was used to treat melanoma cells for $48 \mathrm{~h}$. Cells were assessed for DNA damage by $\gamma \mathrm{H} 2 \mathrm{AX}$ (f). Aged fibroblasts (AG13004 and AG11726) were treated with a-sFRP2 $(15 \mu \mathrm{g} / \mathrm{ml})$ for $72 \mathrm{~h}$ and this conditioned media was used to treat melanoma cells for $48 \mathrm{~h}$. Cells were assessed for DNA damage by $\gamma \mathrm{H} 2 \mathrm{AX}$ (g). 
a

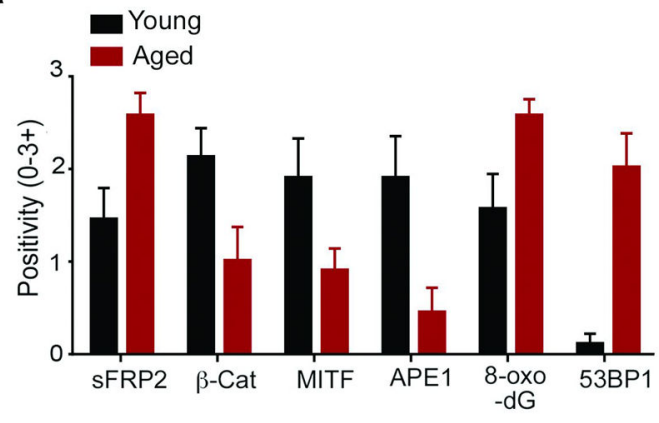

b

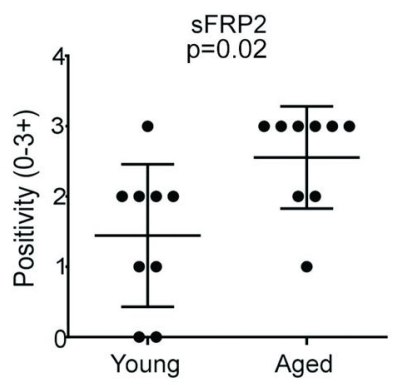

C
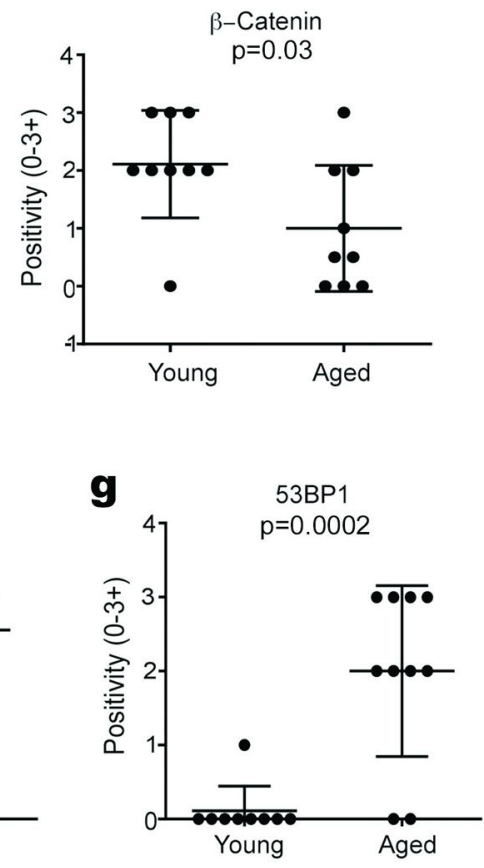

Extended Data 8. Analysis of SFRP2, $\beta$-catenin, MITF, 8-oxo-dG, APE1, and 53BP1 in individual patients

Multiple melanoma samples from aged patients (red bars), and young patients (black bars) were compared. Bars represent average staining intensity (3- highest, 0-lowest) in all patients (n=9/group) for indicated proteins (a). Dot plots of staining intensity (0-3+) in individual patient samples for: sFRP2 (b) $\beta$-catenin (c) MITF (d) APE1 (e), 8-oxodG (f) and 53BP1 (g). pvalues for each graph obtained by Mann Whitney tests. 
a

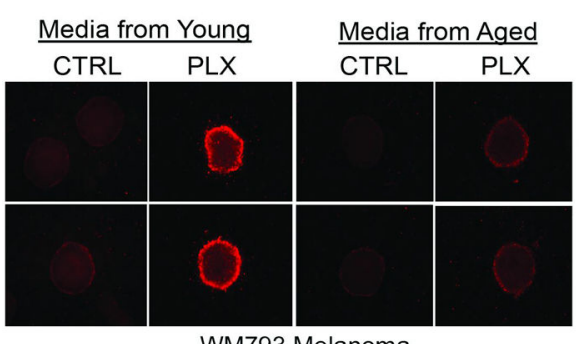

WM793 Melanoma b

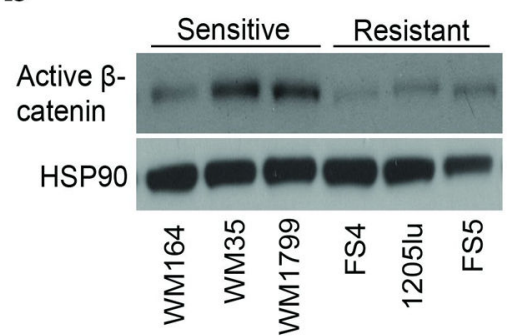

C Yumm 1.7 melanoma

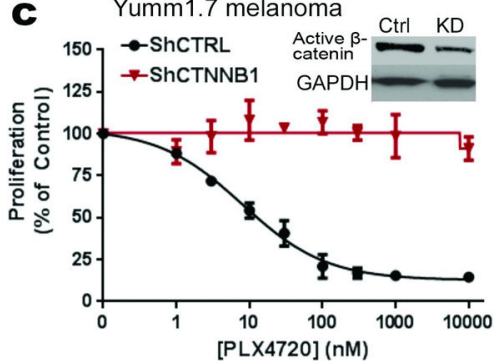

d

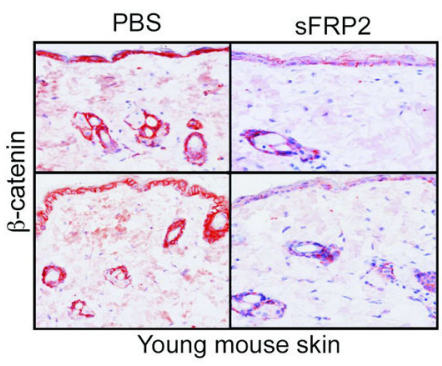

e

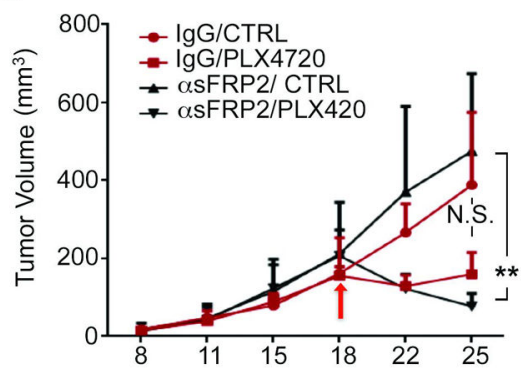

f

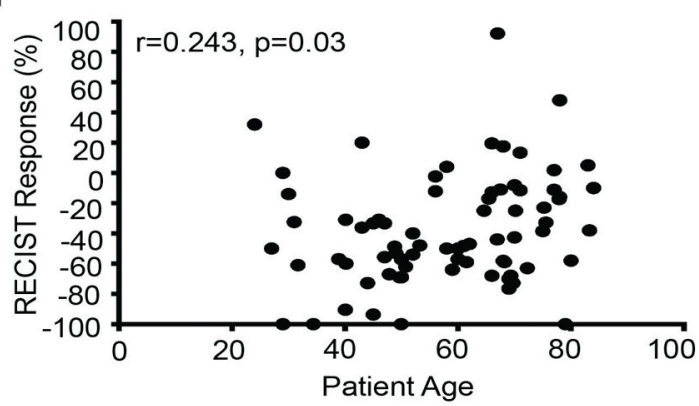

Extended Data 9. $\beta$-catenin predicts for sensitivity to vemurafenib

Melanoma spheroids were embedded in collagen and treated with 1 $\mu \mathrm{M}$ PLX4720 in the presence of conditioned media from either young or aged fibroblasts. After $48 \mathrm{~h}$, spheroids were assessed for cell death by staining with ethidium homodimer (magnification, 40×) (a). In cells intrinsically sensitive to vemurafenib in culture, $\beta$-catenin expression is increased (b). Knockdown of $\beta$-catenin in Yumm1.7 cells decreases their sensitivity to PLX4720. Spearman's correlation between dose and percent proliferation is significant in CTRL cells $[\mathrm{p}<0.0001, \mathrm{r}=-1.000]$ whereas shCTNNB1 cells indicated no significant changes in curve after treatment $[\mathrm{p}=0.948, \mathrm{r}=0.03]$ (c). Young mice ( 8 weeks, $\mathrm{n}=10$ /group) were injected with rsFRP2 (200ng/mL, twice weekly) and skin was examined for $\beta$-catenin levels by immunohistochemistry. Representative images, magnification 200× (d). Yumm1.7 tumors were injected in aged mice pre-treated with a-sFRP2 antibody ( $1 \mathrm{mg} / \mathrm{kg}$, once weekly). Mice were then administered either control or $417 \mathrm{mg} / \mathrm{kg}$ PLX4720 laced chow. Analysis of variance is significant between treatments ( $\mathrm{p}<0.0001)$. Two-tailed unpaired t test using rank sum (Mann Whitney) was performed on tumor volumes on day 25 (1 week after treatment). Results were significant in $\mathrm{SFRP} 2$ treatment $(\mathrm{p}=0.036)$ and insignificant in $\mathrm{IgG} 2 \mathrm{a} \kappa$ treatment $(\mathrm{p}=0.057)$ (e). Patient samples show a continuum of decreased response in relation to age, Spearman's correlation between percent response and age is significant $[r=0.243, p=0.035]$ (f). Data are represented as mean \pm s.d for each graph (c,e).

\section{Extended Data Table 1}

Details Of Skin Fibroblasts Obtained From Healthy Donors.

\begin{tabular}{|l|l|l|l|l|l|}
\hline Line & Sex & Age & Disease State & $\begin{array}{l}\text { Tissue } \\
\text { Type }\end{array}$ & Biopsy Source \\
\hline GM04390 & F & 23 & Healthy & Skin & Arm \\
\hline
\end{tabular}




\begin{tabular}{|l|l|l|l|l|l|}
\hline Line & Sex & Age & Disease State & $\begin{array}{l}\text { Tissue } \\
\text { Type }\end{array}$ & Biopsy Source \\
\hline GM02674 & F & 29 & Healthy & Skin & unspecified \\
\hline AG11732 & F & 24 & Healthy & Skin & Arm \\
\hline AG07720 & F & 24 & Healthy & Skin & Arm \\
\hline AG11735 & F & 26 & Healthy & Skin & Arm \\
\hline AG07124 & F & 26 & Healthy & Skin & Arm \\
\hline TP-113 & M & 27 & Healthy & Skin & Arm \\
\hline 2003-071-056 & M & 32 & Healthy & Skin & Arm \\
\hline GM02673 & M & 33 & Healthy & Skin & unspecified \\
\hline 2003-071-051 & M & 23 & Healthy & Skin & Arm \\
\hline AG04062 & M & 31 & Healthy & Skin & Arm \\
\hline 2003-071-032 & M & 28 & Healthy & Skin & Arm \\
\hline 2003-071-057 & M & 31 & Healthy & Skin & Arm \\
\hline
\end{tabular}

\begin{tabular}{|l|l|l|l|l|l|}
\hline AG08620 & F & 64 & Healthy & Skin & Arm \\
\hline AG11726 & F & 56 & Healthy & Skin & Arm \\
\hline AG08517 & F & 66 & Healthy & Skin & Arm \\
\hline AG11489 & F & 66 & Healthy & Skin & Arm \\
\hline AG07757 & F & 61 & Healthy & Skin & Arm \\
\hline AG08529 & F & 60 & Healthy & Skin & Arm \\
\hline AG08701 & F & 62 & Healthy & Skin & Arm \\
\hline AG08379 & F & 60 & Healthy & Skin & Arm \\
\hline AG12597 & F & 62 & Healthy & Skin & Arm \\
\hline AG12988 & F & 56 & Healthy & Skin & Arm \\
\hline AG12589 & F & 68 & Healthy & Skin & Arm \\
\hline AG09878 & F & 61 & Healthy & Skin & Arm \\
\hline GM13335 & M & 57 & Healthy & Skin & Arm \\
\hline AG13095 & M & 62 & Healthy & Skin & Arm \\
\hline AG09157 & M & 63 & Healthy & Skin & Arm \\
\hline AG11079 & M & 63 & Healthy & Skin & Arm \\
\hline AG10942 & M & 50 & Healthy & Skin & Arm \\
\hline AG06281 & M & 67 & Healthy & Skin & Arm \\
\hline AG13004 & M & 68 & Healthy & Skin & Arm \\
\hline
\end{tabular}

\section{Supplementary Material}

Refer to Web version on PubMed Central for supplementary material.

\section{Acknowledgments}

We thank Dr. Dario Altieri (The Wistar Institute), Dr. Richard Marais (Cancer Research UK Manchester Institute), Dr. Ze'ev Ronai (Sanford Burnham Prebys Medical Discovery Institute), Dr. Martin McMahon (UCSF) and Dr. Bert Vogelstein (Sidney Kimmel, Cancer Center, Johns Hopkins School of Medicine) for critical comments on the 
manuscript. We thank Dr. Rajasekharan Somasundaram (Wistar) for helpful advice on immune analyses, and Dr. Meenhard Herlyn (Wistar) for the WM cell lines, and Dr. Gideon Bollag of Plexxicon for PLX4720. We thank Russell Delgiacco and Dmitry Gourevitch of the Wistar Histology Core, and Fred Keeney of the Wistar Imaging facility, as well as David Schultz of the Molecular Screening Facility. We thank Ms. Ashini Dias-Wanigasekera, Ms. Eleanor Gaddy and Mr. Matthew Ha for technical assistance, and Dr. Rachel Locke for professional editing of the manuscript. This work was supported in part by funds from the Intramural Program of the National Institute on Aging, Baltimore, MD (NM, KGB, RM, WWIII, LF), The Harry J. Lloyd Foundation (KM, ATW), P01 CA 114046-06 (ATW, QL), T32 CA 9171-36 (MRW, CHKIII), an ACS-IRG award (ATW) and RO1 CA174746-01 (ATW, AK). Core facilities at the Wistar are supported by the CCSG grant P30 CA010815.

\section{References}

1. Ruiter D, Bogenrieder T, Elder D, Herlyn M. Melanoma-stroma interactions: structural and functional aspects. Lancet Oncol. 2002; 3:35-43. [PubMed: 11905603]

2. Li G, Satyamoorthy K, Herlyn M. Dynamics of cell interactions and communications during melanoma development. Crit Rev Oral Biol Med. 2002; 13:62-70. [PubMed: 12097238]

3. Hsu MY, Meier F, Herlyn M. Melanoma development and progression: a conspiracy between tumor and host. Differentiation. 2002; 70:522-536. [PubMed: 12492494]

4. Bogenrieder T, Herlyn M. Cell-surface proteolysis, growth factor activation and intercellular communication in the progression of melanoma. Crit Rev Oncol Hematol. 2002; 44:1-15. doi:S1040842801001962 [pii]. [PubMed: 12398996]

5. Dankort D, et al. Braf(V600E) cooperates with Pten loss to induce metastatic melanoma. Nat Genet. 2009; 41:544-552. doi:ng.356 [pii] 10.1038/ng.356. [PubMed: 19282848]

6. Hoek KS. MITF: the power and the glory. Pigment Cell Melanoma Res. 2011; 24:262-263. [PubMed: 21513006]

7. Hoek KS, et al. In vivo switching of human melanoma cells between proliferative and invasive states. Cancer Res. 2008; 68:650-656. doi:10.1158/0008-5472.CAN-07-2491 68/3/650 [pii]. [PubMed: 18245463]

8. Webster MR, Kugel CH 3rd, Weeraratna AT. The Wnts of change: How Wnts regulate phenotype switching in melanoma. Biochim Biophys Acta. 2015; 1856:244-251. [PubMed: 26546268]

9. O'Connell MP, et al. Hypoxia Induces Phenotypic Plasticity and Therapy Resistance in Melanoma via the Tyrosine Kinase Receptors ROR1 and ROR2. Cancer Discov. 2013; 3:1378-1393. doi: 10.1158/2159-8290.CD-13-0005 2159-8290.CD-13-0005 [pii]. [PubMed: 24104062]

10. Flach EH, Rebecca VW, Herlyn M, Smalley KS, Anderson AR. Fibroblasts contribute to melanoma tumor growth and drug resistance. Mol Pharm. 2011; 8:2039-2049. [PubMed: 22067046]

11. Campisi J. The role of cellular senescence in skin aging. J Investig Dermatol Symp Proc. 1998; 3:1-5.

12. Coppe JP, Desprez PY, Krtolica A, Campisi J. The senescence-associated secretory phenotype: the dark side of tumor suppression. Annu Rev Pathol. 2010; 5:99-118. [PubMed: 20078217]

13. Park HW. Biological aging and social characteristics: gerontology, the Baltimore city hospitals, and the national institutes of health. J Hist Med Allied Sci. 2013; 68:49-86. doi:10.1093/jhmas/jrr048 jrr048 [pii]. [PubMed: 23229081]

14. Arozarena I, et al. In melanoma, beta-catenin is a suppressor of invasion. Oncogene. 2011; 30:4531-4543. doi:10.1038/onc.2011.162 onc2011162 [pii]. [PubMed: 21577209]

15. Chien AJ, et al. Activated Wnt/beta-catenin signaling in melanoma is associated with decreased proliferation in patient tumors and a murine melanoma model. Proc Natl Acad Sci U S A. 2009; 106:1193-1198. doi:0811902106 [pii] 10.1073/pnas.0811902106. [PubMed: 19144919]

16. Bouchlaka MN, et al. Aging predisposes to acute inflammatory induced pathology after tumor immunotherapy. J Exp Med. 2013; 210:2223-2237. doi:10.1084/jem.20131219 jem.20131219 [pii]. [PubMed: 24081947]

17. Lento $\mathrm{W}$, et al. Loss of beta-catenin triggers oxidative stress and impairs hematopoietic regeneration. Genes Dev. 2014; 28:995-1004. [PubMed: 24788518] 
18. Widlund HR, et al. Beta-catenin-induced melanoma growth requires the downstream target Microphthalmia-associated transcription factor. J Cell Biol. 2002; 158:1079-1087. [PubMed: 12235125]

19. Liu F, Fu Y, Meyskens FL Jr. MiTF regulates cellular response to reactive oxygen species through transcriptional regulation of APE-1/Ref-1. J Invest Dermatol. 2009; 129:422-431. [PubMed: 18971960]

20. Corazao-Rozas P, et al. Mitochondrial oxidative stress is the Achille's heel of melanoma cells resistant to Braf-mutant inhibitor. Oncotarget. 2013; 4:1986-1998. [PubMed: 24161908]

21. Yu L, et al. Involvement of superoxide and nitric oxide in BRAF inhibitor PLX4032-induced growth inhibition of melanoma cells. Integrative biology : quantitative biosciences from nano to macro. 2014

22. Biechele TL, et al. Wnt/beta-catenin signaling and AXIN1 regulate apoptosis triggered by inhibition of the mutant kinase BRAFV600E in human melanoma. Sci Signal. 2012; 5:ra3. doi: 10.1126/scisignal.2002274 5/206/ra3 [pii]. [PubMed: 22234612]

23. Konieczkowski DJ, et al. A melanoma cell state distinction influences sensitivity to MAPK pathway inhibitors. Cancer Discov. 2014; 4:816-827. [PubMed: 24771846]

24. Yun J, et al. Vitamin C selectively kills KRAS and BRAF mutant colorectal cancer cells by targeting GAPDH. Science. 2015

25. Issa JP. CpG-island methylation in aging and cancer. Current topics in microbiology and immunology. 2000; 249:101-118. [PubMed: 10802941]

26. Issa JP. Aging and epigenetic drift: a vicious cycle. J Clin Invest. 2014; 124:24-29. [PubMed: 24382386]

27. Shah PP, et al. Lamin B1 depletion in senescent cells triggers large-scale changes in gene expression and the chromatin landscape. Genes Dev. 2013; 27:1787-1799. [PubMed: 23934658]

28. Viros A, et al. Ultraviolet radiation accelerates BRAF-driven melanomagenesis by targeting TP53. Nature. 2014; 511:478-482. [PubMed: 24919155]

29. Bald T, et al. Ultraviolet-radiation-induced inflammation promotes angiotropism and metastasis in melanoma. Nature. 2014; 507:109-113. doi:10.1038/nature13111 nature13111 [pii]. [PubMed: 24572365]

30. Berking C, Herlyn M. Human skin reconstruct models: a new application for studies of melanocyte and melanoma biology. Histol Histopathol. 2001; 16:669-674. [PubMed: 11332722] 


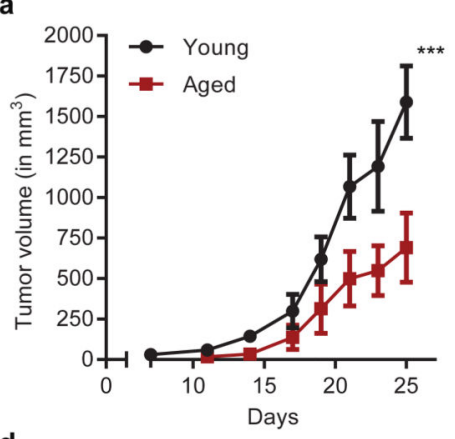

d
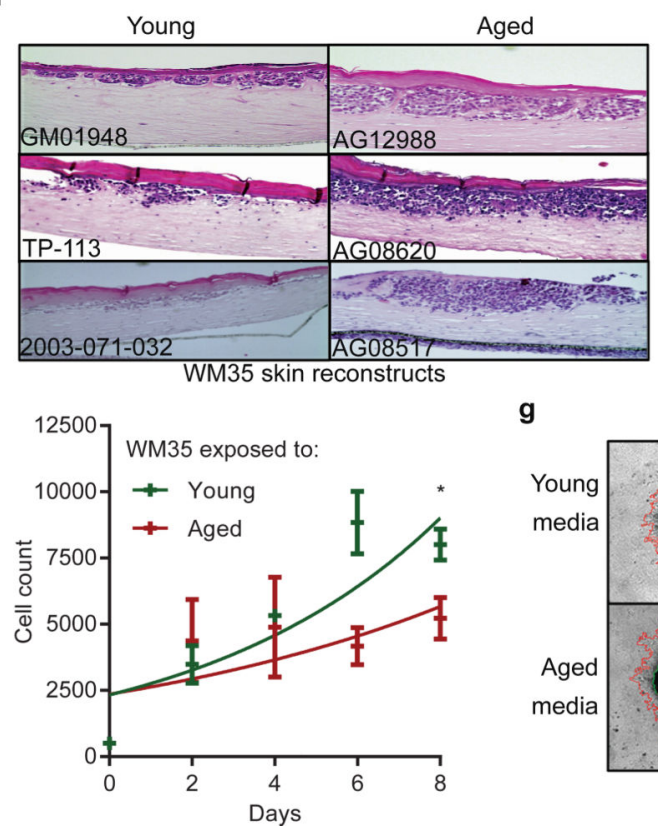

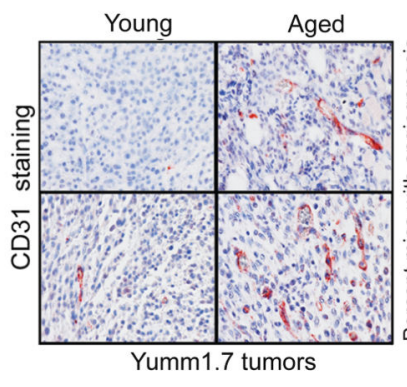

C
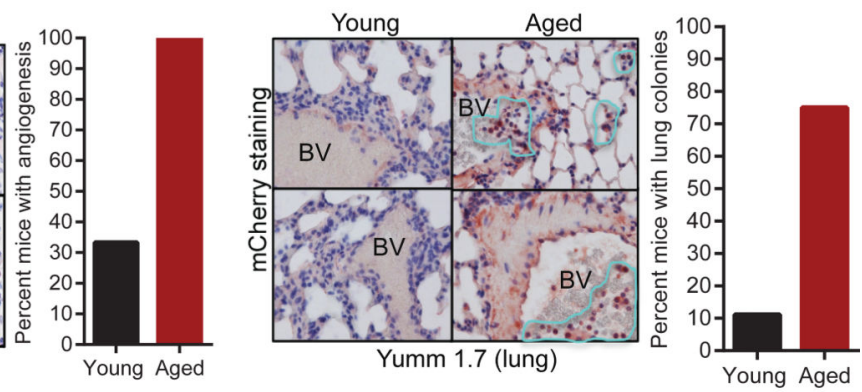

e

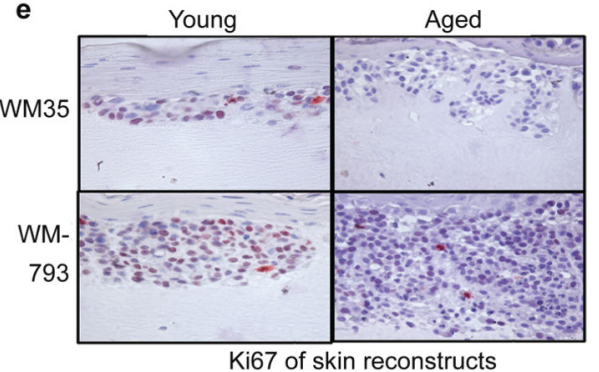

h
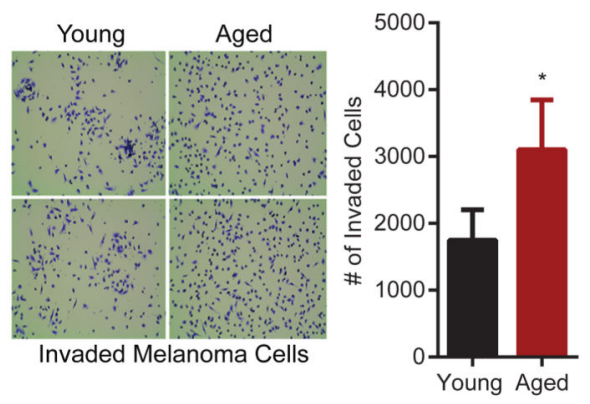

Figure 1. The aged microenvironment promotes phenotype switching

Growth of $1 \times 10^{6}$ YUMM1.7_mCherry cells subcutaneously injected in young ( 8 weeks, $\mathrm{n}=10$ ) and aged (52 weeks, $\mathrm{n}=10$ ) C57/BL6 mice. (ANOVA $\mathrm{p}<0.0001$; Multiple comparison's test using Bonferroni correction, $\mathrm{p}<0.0001$ after day 19)(a). CD31 expression from (a) (magnification 200x). Graph indicates percent of mice with extensive angiogenesis (multiple CD31+ vessels/ field) (b). mCherry staining in mouse lungs (positive cells circled in yellow; magnification 400x) (c). WM35 melanoma cells in skin reconstructs built with young or aged fibroblasts $(n=3$, magnification $150 \times$ ). Invasion was quantified using ImageJ (ANOVA p=0.0006) (d). Ki67 staining from (d) (magnification 400x) (e). Proliferation of WM35 melanoma cells in conditioned media from young or aged fibroblasts $(\mathrm{n}=4$ fibroblasts/group) ANOVA, $\mathrm{p}=0.004$; Holm Sidak correction [day 6 ( $\mathrm{p}=0.002)$, day 8 $(\mathrm{p}=0.034)]$ ( $\mathbf{f})$. Invasion of melanoma spheroids exposed to aged or young conditioned media ( $\mathrm{n}=2$ fibroblasts/group, magnification 40x, two-tailed unpaired t-test, $\mathrm{p}=0.041$ ) (g). Boyden chamber invasion of FS5 melanoma cells treated with young vs. aged fibroblast media (magnification 150x, two-tailed unpaired t-test, $\mathrm{p}=0.043)(\mathbf{h})$. Data represented as mean \pm s.d. (a,d,g,h). 
a

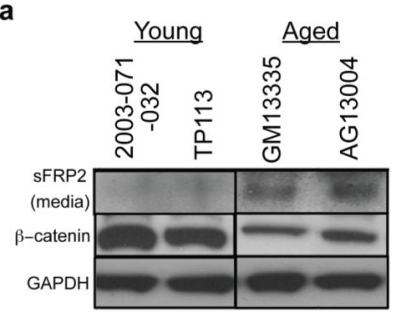

b

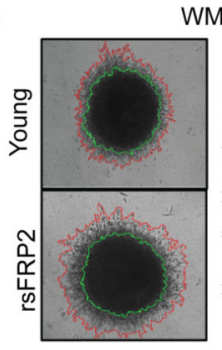

VM35
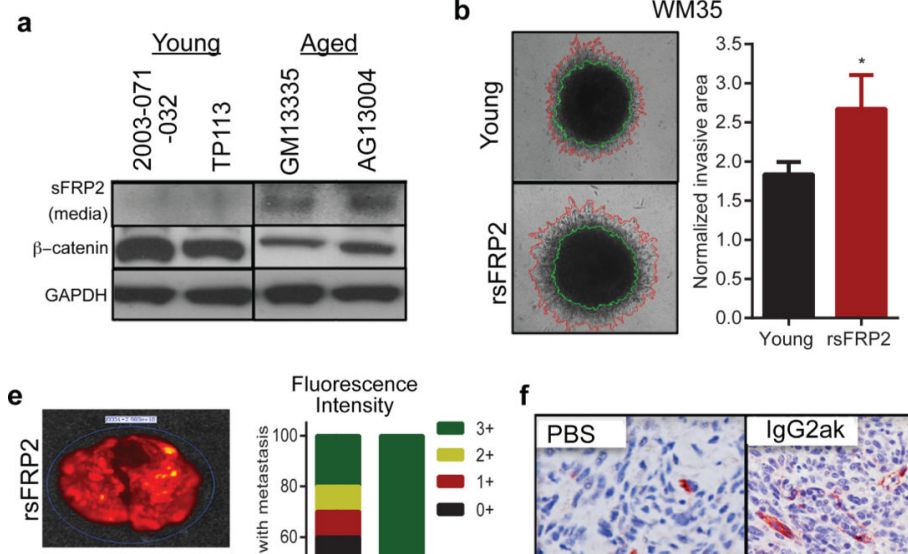

Fluorescence
Intensity
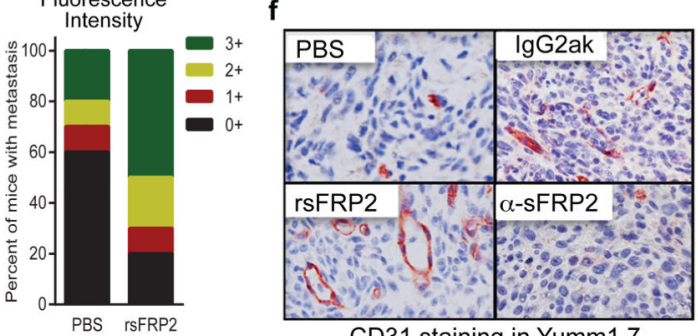

CD31 staining in Yumm1.7

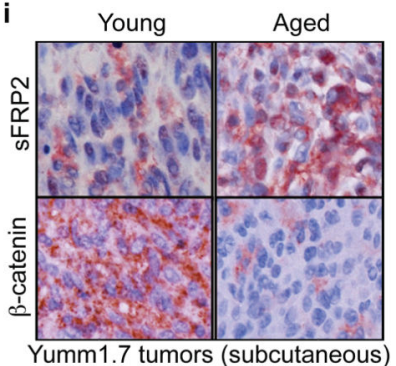

j

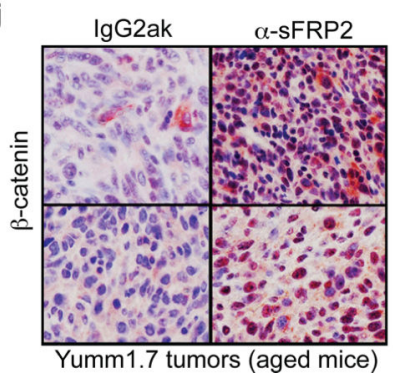

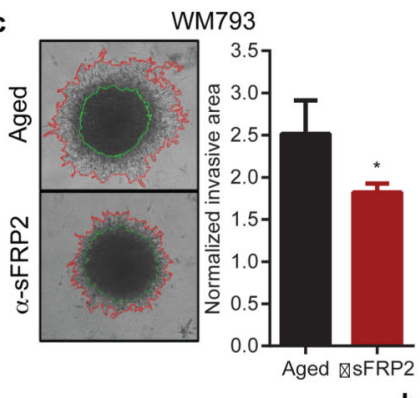

g

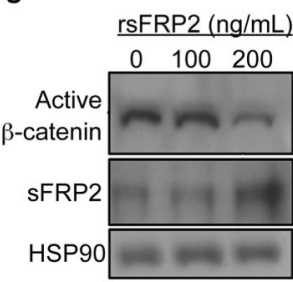

k

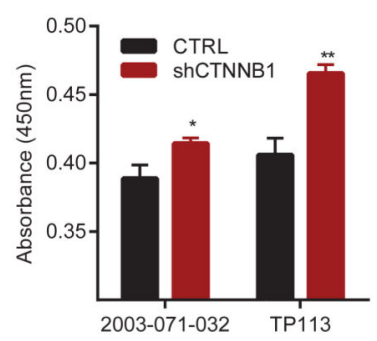

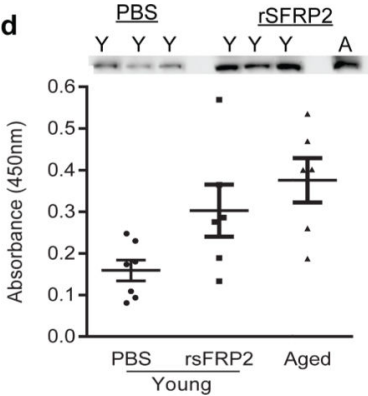

h $\quad$-catenin staining
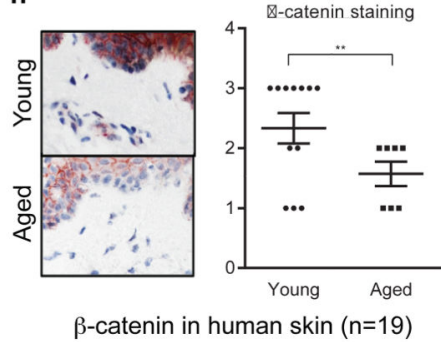

I

$\beta$-catenin in human skin $(n=19)$

Fibroblast Media

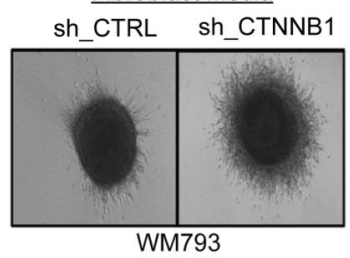

Figure 2. sFRP2 promotes metastasis during aging

Western analysis of sFRP2 (media) and $\beta$-catenin (cell lysates) in fibroblasts (a). Invasion of melanoma spheroids (magnification 40x), treated with rsFRP2 (200ng/ml, 48h, two-tailed unpaired t-test, $\mathrm{p}=0.046)(\mathbf{b})$. Melanoma spheroids treated with media from aged fibroblasts pre-treated with either IgG2aא, or a-sFRP2 monoclonal antibody $(15 \mu \mathrm{g} / \mathrm{ml}, 48 \mathrm{~h}$, magnification $40 \times$, two-tailed unpaired t-test, $\mathrm{p}=0.023)(\mathbf{c})$. $\mathrm{sFRP} 2$ in mouse serum $(\mathrm{n}=10$ / group): young, aged, and young $+200 \mathrm{ng} / \mathrm{mL}$ rsFRP2. ANOVA $(\mathrm{p}=0.015)$, two-tailed unpaired t-test [PBS vs rsFRP2 ( $\mathrm{p}=0.045)$, young vs aged mice $(\mathrm{p}=0.007)](\mathbf{d}) .1 \times 10^{6}$ mCherry labeled Yumm 1.7 cells were injected intravenously into PBS or rsFRP2 ( $200 \mathrm{ng} / \mathrm{mL}$ ) treated young mice (6-8 weeks, $\mathrm{n}=10$ /group). Graph indicates percent positive lungs recorded as IVIS fluorescence intensity where highest intensity is $3+($ red), i.e., multiple metastatic foci, 0-lowest intensity, i.e., no metastases (green) (e). Yumm 1.7 tumors in young mice (6-8 weeks, $\mathrm{n}=10$ /group) treated with either PBS or rsFRP2 $(200 \mathrm{ng} / \mathrm{mL})$ or in aged mice ( 52 weeks, $\mathrm{n}=5$ /group) treated with either control IgG2ak, or a-sFRP2 monoclonal antibody $(1 \mathrm{mg} / \mathrm{kg}$ ) were assessed for CD31 (magnification 400×) (f). Western analysis of non-phosphorylated (active) $\beta$-catenin in melanoma cells treated with rsFRP2 for $48 \mathrm{~h}$ (g). $\beta$-catenin expression in young and aged human skin ( $<35 \mathrm{yr}, \mathrm{n}=12 ;>55 \mathrm{yr}, \mathrm{n}=7)$. Slides were scored for positivity (3-highest, 0 -lowest), magnification $400 \times$, unpaired t-test using rank-sum $(\mathrm{p}=0.019)(\mathbf{h})$. sFRP2 and $\beta$-catenin in Yumm1.7 tumors in young and aged mice (magnification 600x) (i). Aged tumor-bearing mice ( $>52$ weeks, $n=5 /$ group) treated 
with a-sFRP2 monoclonal antibody $(1 \mathrm{mg} / \mathrm{kg})$. Tumors were stained for $\beta$-catenin (magnification 400x) (j). sFRP2 ELISA in shCTNNB1 vs. shCTRL fibroblast media. Twotailed unpaired t-test [2003-071-032 ( $\mathrm{p}=0.015)$, TP113 $(\mathrm{p}=0.008)$ ] $(\mathbf{k})$. Invasion of melanoma spheroids treated with TP113 shCTNNB1 conditioned media for $48 \mathrm{~h}$ (magnification 40×) (l). Data represented as mean \pm s.d. (b,c,d,h,k). 


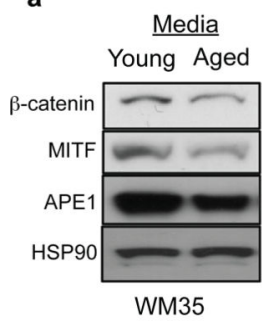

e

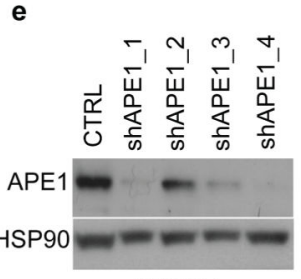

WM164

g

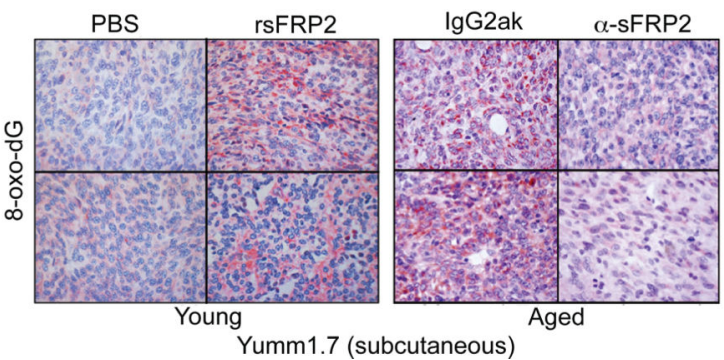

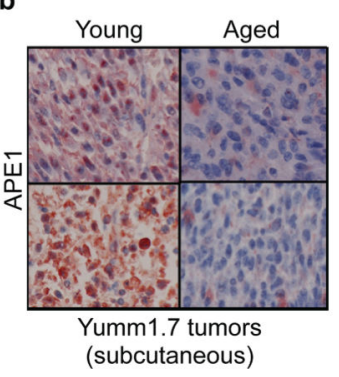

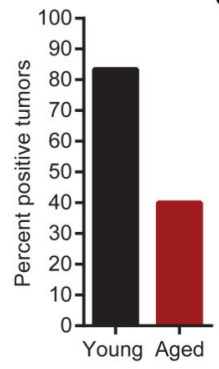

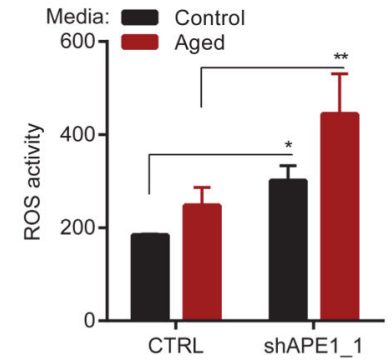

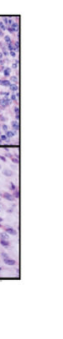

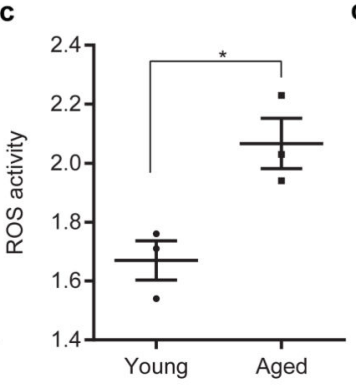

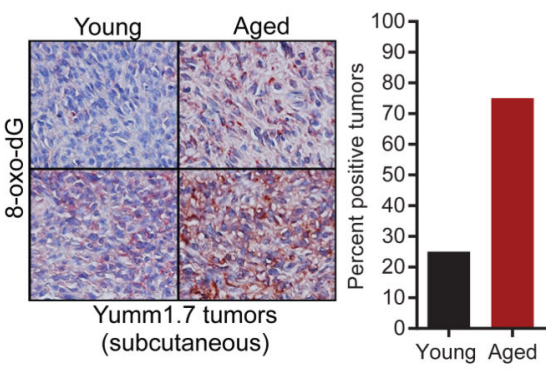

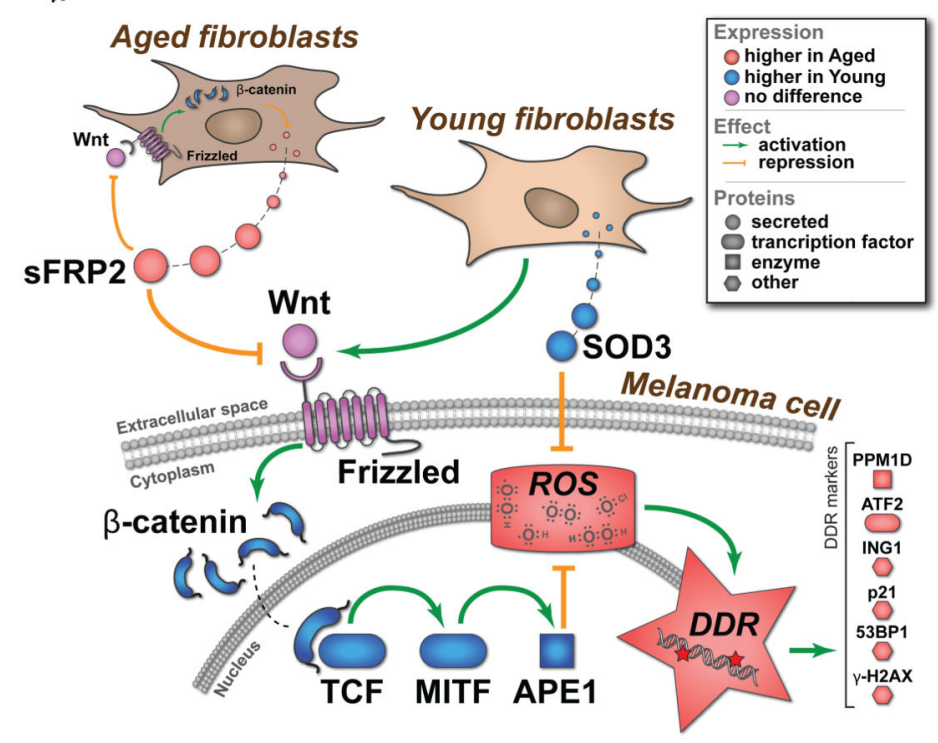

Figure 3. Loss of APE1 renders melanoma cells more sensitive to oxidative stress in an aged microenvironment

$\beta$-catenin, APE1 and MITF in melanoma cells exposed to aged or young fibroblast media (a). APE1 in Yumm1.7 tumors in aged and young mice, percent positive tumors is quantified (magnification 400×) (b). ROS levels in multiple young and aged fibroblasts (two-tailed unpaired t-test, $\mathrm{p}=0.022$ ) (c). Percent positive 8-oxo-dG Yumm1.7 tumors implanted in aged and young mice (magnification 400x) (d). Western analysis of WM35 melanoma cells after APE1 knockdown (e). ROS levels in shAPE1 melanoma cells in absence (two-tailed t-test, $\mathrm{p}=0.012$ ), and presence (two-tailed $\mathrm{t}$-test, $\mathrm{p}=0.008$ ) of conditioned media from aged fibroblasts (f). 8-oxo-dG levels assessed in tumors from young mice treated with either PBS or rsFRP2 and aged mice treated with either IgG2ak or a-sFRP2 (magnification 400x). (g). Schematic of sFRP2 effects in melanoma cells exposed to aged or young fibroblasts (h). Data represented as mean \pm s.d. (c,f). 

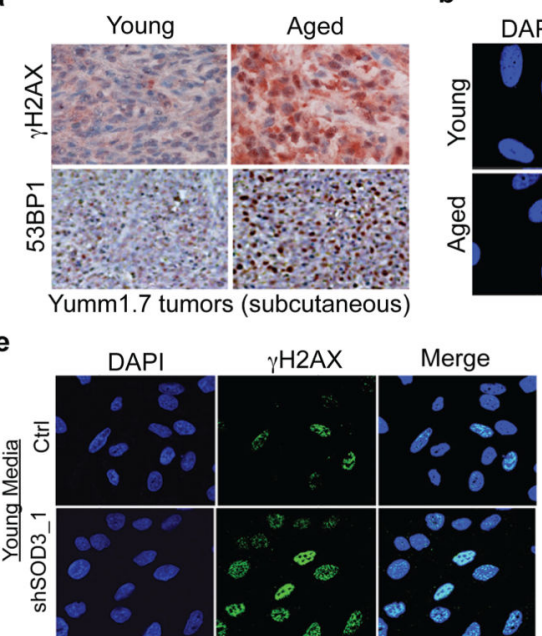

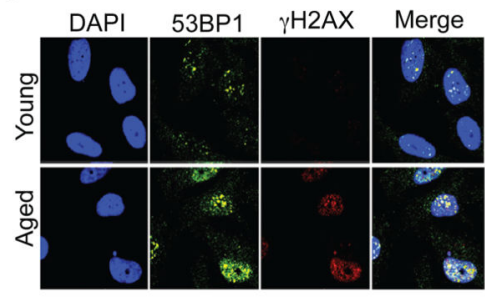

f

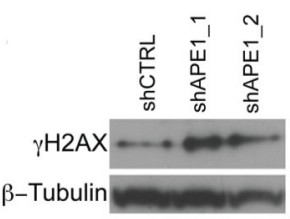

WM164

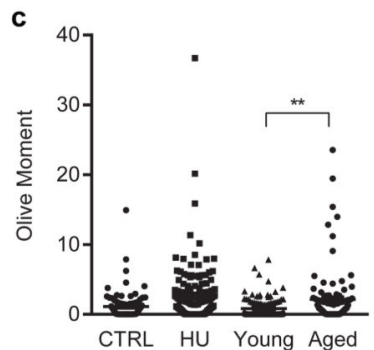

g

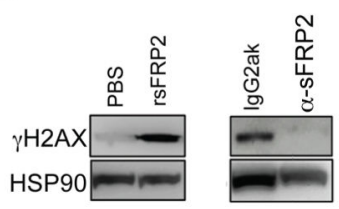

WM793 d

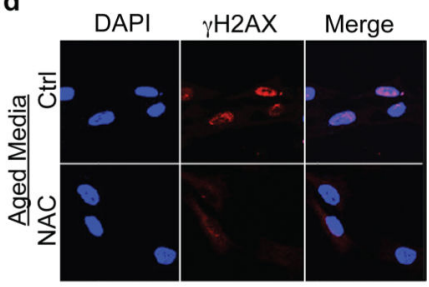

h Yumm1.7 tumors

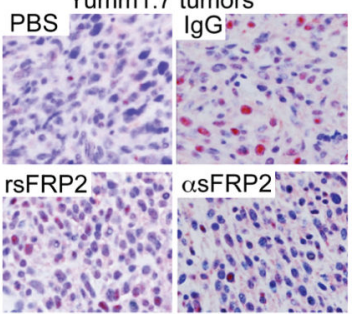

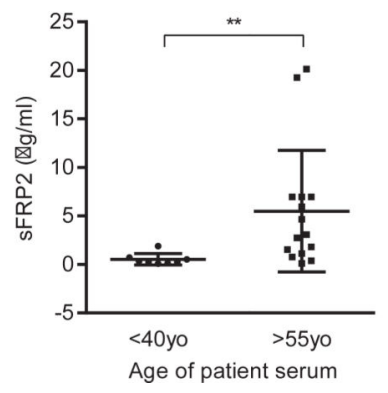

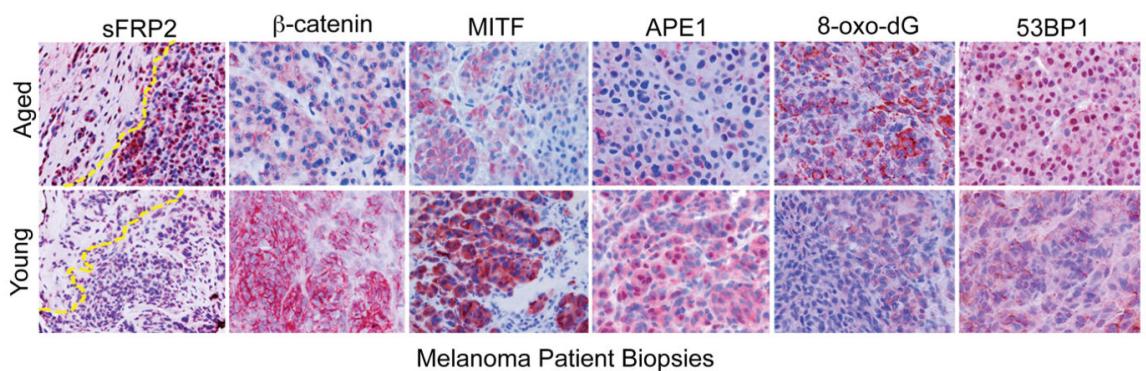

Figure 4. Melanoma cells in an aged microenvironment exhibit increased DNA damage markers $\gamma \mathrm{H} 2 \mathrm{AX}$ and 53BP1 levels in Yumm1.7 tumors in aged and young mice (magnification $400 \times$ ) (a), and in melanoma cells treated with conditioned media from young and aged fibroblasts (b). Comet assay analysis in melanoma cells treated with conditioned media from aged and young fibroblasts. Two-tailed unpaired t-test with Welch's correction (young vs aged, $\mathrm{p}=0.002)$. Data represented as mean \pm s.e.m. (c). $\gamma \mathrm{H} 2 \mathrm{AX}$ in melanoma cells treated with conditioned media from aged fibroblasts pretreated with 20mM NAC (IF; 48h) (d), conditioned medium from young fibroblasts with SOD3 knock-down (IF; 72h) (e), or in melanoma cells with APE1 knockdown (Western; 48h) (f). $\gamma \mathrm{H} 2 \mathrm{AX}$ in melanoma cells exposed for $48 \mathrm{~h}$ to conditioned media from young fibroblasts pre-treated with rsFRP2 or aged fibroblasts treated with a-sFRP2 antibody $(72 \mathrm{~h})(\mathrm{g}) .53 \mathrm{BP} 1$ in tumors from young mice treated with PBS or rsFRP2 and aged mice treated with IgG2ak or a-sFRP2 (magnification 600x) (h). Serum sFRP2 ELISA in young and aged melanoma patients ( $N=8$ young, $N=15$ aged; unpaired t-test with Welch's correction, $\mathrm{p}=0.008$ ). Data represented as mean \pm s.d. (i). Representative images from one young and aged patient for indicated proteins in the proposed pathway (magnification 400x) (j). 
a

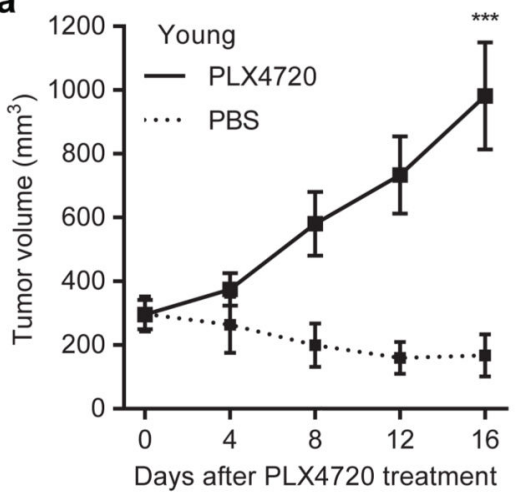

b

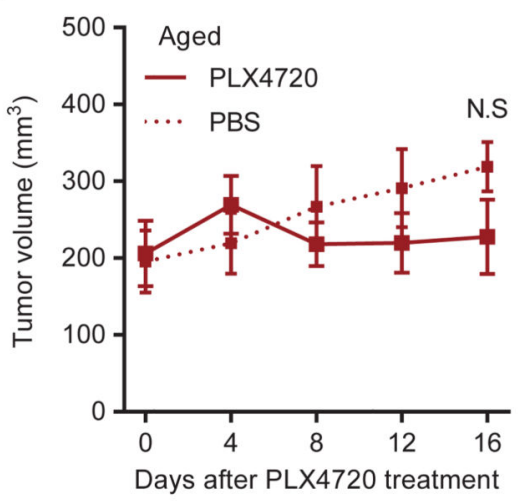

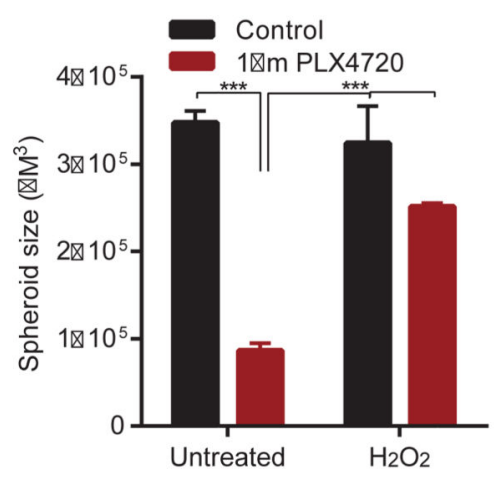

d

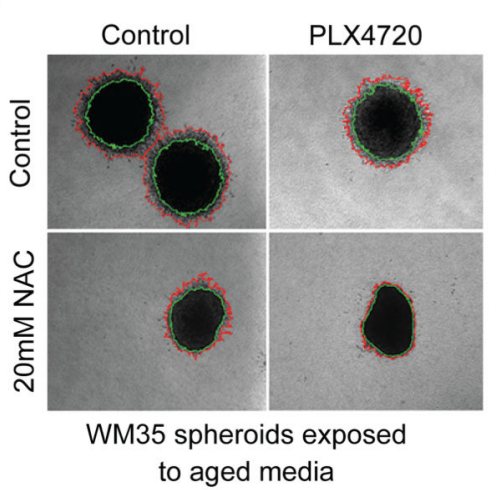

h

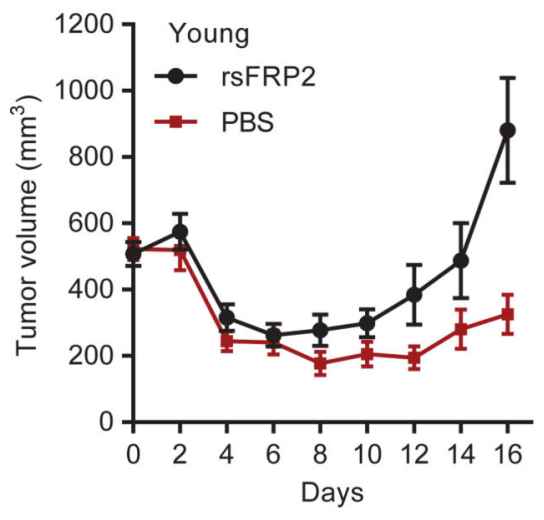

e

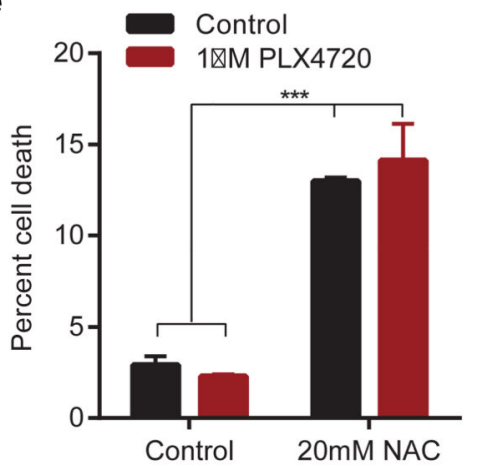

i

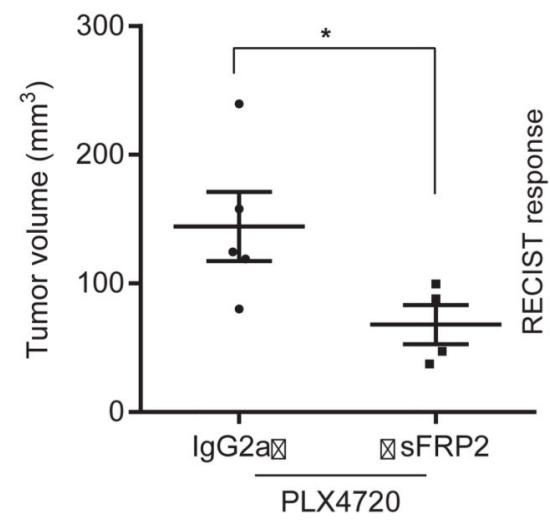

f

j

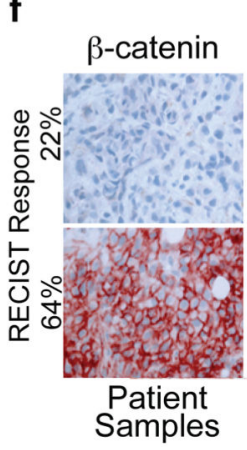

g
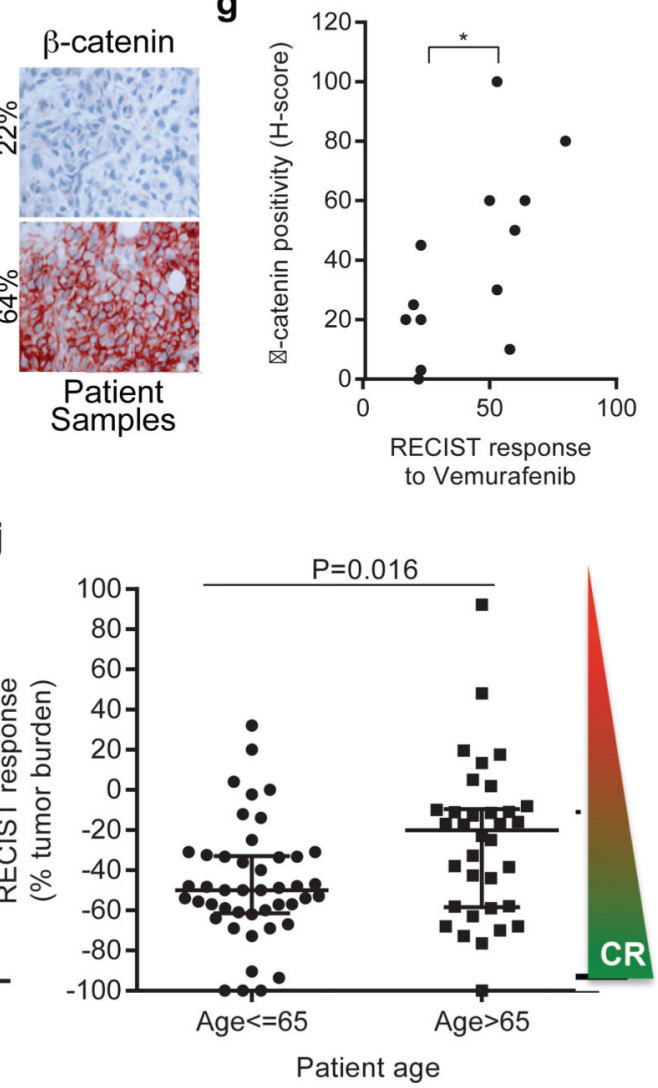

Figure 5. The aged microenvironment induces therapy resistance

Yumm 1.7 tumors in young ( 8 weeks, $n=10$ mice/treatment) or aged ( 52 weeks, $n=10$ mice/ treatment) mice fed PLX4720 (417 mg/kg) chow. Tumors responded in young $\left(\mathrm{p}=6 \times 10^{-5}\right.$, two way paired t-test) $(\mathbf{a})$, but not aged mice $(\mathrm{p}=0.361)$. Dotted lines indicate untreated controls (b). Melanoma spheroids were treated with $100 \mathrm{nM} \mathrm{H}_{2} \mathrm{O}_{2}(48 \mathrm{~h})$, embedded in collagen and treated with young media containing $1 \mu \mathrm{M}$ PLX4720 (ANOVA, $\mathrm{p}<0.0001$ ) (c). WM35 spheroids were embedded in collagen and treated with aged media containing $1 \mu \mathrm{M}$ PLX4720 and/or 20mM NAC. Invasion was measured after 48h (representative images, magnification 40×, one-way ANOVA, $\mathrm{p}=0.02$ ). 20mM NAC (unpaired t-test, $\mathrm{p}=0.03$ ) and $1 \mu \mathrm{M}$ PLX4720 (two-tailed unpaired t-test, $\mathrm{p}=0.006$ ) compared to control (d). Live-dead 
staining of spheroids from (d). ANOVA, $\mathrm{p}<0.0001$; Holm-Sidak's multiple comparisons test indicated $(\mathrm{p}<0.0001)$ after pre-treatment with $20 \mathrm{mM}$ NAC in presence of either control or $1 \mu \mathrm{M}$ PLX4720 (e). $\beta$-catenin staining of biopsies (magnification $200 \times$ ) from patients undergoing vemurafenib treatment (percentage indicates percent RECIST response) (f). Tabulation of patient samples correlating H-score (intensity of stain per field of cells) to RECIST response (two-tailed paired t-test, $\mathrm{p}=0.035$ ). $30 \%$ is considered a responder by RECIST criteria (g). Young mice (n=10/group) with Yumm1.7 tumors were treated with rsFRP2 (200ng/mL) bi-weekly. PLX4720 (417/mg/kg) was administered once the tumor reached $500 \mathrm{~mm}^{3}$. rsFRP2-treated (red line) vs control (blue line) young mice (ANOVA, $\mathrm{p}=0.009$; Holm-Sidak corrected multiple comparisons, $\mathrm{p}<0.05$ after day 12 , mean \pm s.e.m.) (h). Yumm1.7 tumors were injected in aged mice ( 52 weeks, $n=5 /$ group) pre-treated with asFRP2 antibody ( $1 \mathrm{mg} / \mathrm{kg}$, once weekly). Mice were administered control or $417 \mathrm{mg} / \mathrm{kg}$ PLX4720-laced chow. Tumor volume at day 25 is shown (unpaired t-test with Welch's correction, $\mathrm{p}=0.048$ ). (i). RECIST response in patients under 65 vs. over 65 . Two sample Wilcoxon rank-sum (Mann Whitney) test indicated statistical significance $(\mathrm{p}=0.016)(\mathbf{j})$. Data represented as mean \pm s.d. (a,b,c,e,i,j, $)$. 